\title{
CLOSED AND OPEN CONFORMAL FIELD THEORIES AND THEIR ANOMALIES
}

\author{
PO HU AND IGOR KRIZ
}

\section{INTRODUCTION}

The main purpose of this paper is to give rigorous mathematical foundations for investigating closed and closed/open conformal field theories (CFT's) and their anomalies. In physics, the topic of closed/open CFT has been extensively discussed in the literature (see e.g. 8, 19, 20, 7]). Our investigation was originally inspired by two sources: Edward Witten (cf. 35) proposed a general program for using $K$-theory to classify stable $D$-branes in string theory. On the other hand, Moore and Segal 22] obtained a mathematically rigorous approach to classifying $D$-branes in the case of 2-dimensional topological quantum field theory (TQFT).

We attempted to consider a case in between, namely $D$-branes in conformal field theory. There are many reasons for $D$-branes to be easier in CFT than in string theory. First of all, one does not have to insist on anomaly cancellation, and can investigate the anomaly instead. Another reason is that CFT makes good sense even without supersymmetry, while string theory does not. (In fact, in this paper, we restrict attention to non-supersymmetric CFT, although mostly just in the interest of simplicity.) Most importantly, however, any CFT approach to string theory amounts to looking at the complicated string moduli space only through the eyes of one tangent space, which is a substantial simplification. We will make some comments on this relationship between CFT and string theory in Section 3 below.

On the other hand, CFT is incomparably more complicated than 2-dimensional TQFT. Because of this, in fact, a theorem classifying $D$-branes (as outlined in 22 for TQFT) seems, at the present time, out of reach for CFT. However, an exact mathematical definition of the entire structure of closed/open CFT is a reasonable goal which we do undertake here. To this end, we use our formalism of stacks of lax commutative monoids with cancellation (SLCMC's), developed in [14, and reviewed in the Appendix. Also, we describe analogues of some of the concepts of 22 for CFT's, and observe some interesting new phenomena. For example, the correct generalization of module over the algebra corresponding to TQFT, is not a VOA module, but a different kind of module (which we call $D$-brane module). When specializing to genus 0 surfaces, this leads to a generalization of the notion of module over an operad. The $K$-theory of such modules (with some finiteness assumption) is therefore a candidate for a CFT analogue of the $K$-theory considered in 22] (CFT " $D$-brane cohomology"). These topics are discussed in Section 2 below. 
In Section 4 we give a basic example, the free bosonic CFT (=linear $\sigma$-model), and show how to obtain the $D$-brane modules corresponding to Von Neumann and Dirichlet boundary conditions for open strings. As we will see, however, even in this basic case, a substantial complication is giving a mathematically rigorous treatment of the convergence issues of the CFT.

Up to this point, we suppressed the discussion of anomaly, by assuming that anomaly is 1-dimensional. However, there is an obvious suggestion a parallel between the set of $D$-branes of a closed/open CFT, and the set of labels of a modular functor of a rational CFT (RCFT, see 23, 24, 25, 29]). It therefore seems we should look for axioms for the most general possible kind of anomaly for closed/open CFT, which would include sets of both $D$-branes and modular functor labels.

There are, however, further clues which suggests that the notion of "sets" in this context is too restrictive. Notably, the free $\mathbb{C}$-vector space $\mathbb{C} S$ on the set of labels $S$ of a modular functor is the well known Verlinde algebra 34. But the multiplication rule of the Verlinde algebra uses only of dimensions of vector spaces involved in the modular functor, so it seems that if one wants to consider the spaces themselves, it is that one should consider, instead of $\mathbb{C} S$, the free 2-vector space on $S$ ). Is it possible to axiomatize modular functors for RCFT's in a way which uses 2-vector spaces in place of sets of labels?

In Section [5] we answer this last question in the affirmative. This is rather interesting, because it leads to other questions: the authors [14 previously proposed RCFT as a possible tool for geometrically modelling elliptic cohomology, while Baas-Dundas-Rognes 2] obtained a version of elliptic cohomology based on 2-vector spaces. Is there a connection? Observations made in 2] show that the right environment of such discussion would be a suitable group completion of the symmetric bimonoidal category $\mathbb{C}_{2}$ of vector spaces, while simultaneously noting the necessary difficulty of any such construction. Nevertheless, we propose in, Section [6] such group completion, despite major technical difficulties. Our construction involves super-vector spaces, and thus suggests further connections with P. Deligne's observation [16 that the modulars functor of $b c$-systems must be considered as super-vector spaces, and with the work of Stolz-Teichner 32, who, in their approach to elliptic objects, also noticed the role of fermions and, in effect, what amounts 1-dimensional super-modular functors.

However, let us return to $D$-branes. Is it possible to formulate axioms for anomalies of closed/open CFT's analogous to the 2-vector space approach to modular functors? We give, again, an affirmative answer, although another surprise awaits us here: while the "set of labels" of a modular functor was naturally a 2-vector space, the "set of $D$-branes" of a closed/open CFT must be a 3-vector space! We discuss this, and propose axioms for a general anomaly of closed/open CFT in Section 7. An intriguing question is whether the group completion approach of Section 6 can also be extended to the case closed/open CFT anomaly.

Acknowledgement: The authors would like to think N.A.Baas and J.Rognes for their helpful comments, and for detecting mistakes in the original version of this paper. 


\section{Closed/open CFT's with 1-dimensional anomaly, $D$-Brane modules AND $D$-BRANe COHOMOLOGY}

There is substantial physical literature on the subject of $D$-branes (see e.g. 8 19, 20, 7]). In this paper, we shall discuss a mathematically rigorous approach to $D$-branes in non-supersymmetric CFT's. Moreover, in Sections 24] we shall restrict attention to 1-dimensional anomaly allowed both on the closed CFT and the $D$-brane. More advanced settings will be left to the later sections.

We begin by defining the stack of lax commutative monoids with cancellation (SLCMC) corresponding to oriented open/closed string (more precisely conformal field) theory. SLCMC's were introduced in 14, but to make this paper selfcontained, we review all the relevant definitions in the Appendix. We consider a set $L$. This is not our set of labels, it is the set of $D$-branes. Our set of labels consists of $K^{\prime}=L \times L$ which we will call open labels and we will put $K=K^{\prime} \amalg\{1\}$ where 1 is the closed label.

To define the SLCMC $\mathcal{B}$ for oriented open/closed CFT, we shall first define the LCMC of its sections over a point. As usual (see [14]), the underlying lax commutative monoid is the category of finite sets labelled by a certain set $K$, not necessarily finite. Before describing the exactly correct analytic and conformal structure, we first specify that these are compact oriented surfaces (2-manifolds) $X$ together with homeomorphic embeddings $c_{i}: S^{1} \rightarrow \partial X, d_{j}: I \rightarrow \partial X$ with disjoint images. Moreover, each $c_{i}$ is labelled with 1 , and each $d_{j}$ is labelled by one of the open labels $K^{\prime}$. Moreover, each connected component of

$$
\partial X-\bigcup \operatorname{Im}\left(c_{i}\right)-\bigcup \operatorname{Im}\left(d_{j}\right)
$$

(which we shall call $D$-brane components) is labelled with an element of $L$, and each $d_{j}$ is labelled with the pair $\left(\ell_{1}, \ell_{2}\right) \in L \times L$ of $D$-branes which the beginning point and endpoint of $d_{j}$ abut. The $c_{i}$ 's and $d_{j}$ 's are considered inbound or outbound depending on the usual comparison of their orientation with the orientation of $X$.

It is now time to describe the smoothness and conformal structure on $X$. To this end, we simply say that $X$ is a smooth complex 1-manifold with analytic (real) boundary and corners; this means that, the interior of $X$ is a complex 1-manifold, and the neighbourhood of a boundary point $x$ of $X$ is modeled by a chart which whose source is either an open subset of the halfplane $\mathbb{H}=\{z \in \mathbb{C} \mid \operatorname{Im}(z) \geq 0\}$ where 0 maps to $x$ or an open subset of the quadrant $\mathbb{K}=\{z \in \mathbb{C} \mid \operatorname{Im}(z) \geq 0, \operatorname{Re}(z) \geq 0\}$ where 0 maps to $x$; the transition maps are (locally) holomorphic maps which extend biholomorphically to an open neighbourhood of 0 in $\mathbb{C}$. The points of the boundary whose neighbourhoods are modelled by open neighborhoods of 0 in $\mathbb{K}$ are called corners. Further, we specify exactly which points are the corners of an open/closed string world sheet: we require that the corners be precisely the endpoints of the images of open string boundary parametrizations. We also require that open as well as closed string parametrizations be real-analytic diffeomorphisms onto their image; this completes the definition of the objects.

An isomorphism $X \rightarrow Y$ is a diffeomorphism which preserves complex structure, $D$-brane labels, is smooth on the interior, and commutes with the $c_{i}, d_{j}$ (which 
we shall call parametrization components - note that the set of parametrization components is not ordered, so an automorphism may switch them).

Now to define the SLCMC $\mathcal{B}$, the main issue is fixing the Grothendieck topology. We use simply finite-dimensional smooth manifolds with open covers. As usual, the underlying stack of lax commutative monoids is the stack of covering spaces with locally constant $K$-labels (analogously to [14]). Sections of $\mathcal{B}$ over $M$ are smooth manifolds fibered over $M$, where the fibers are elements of $\mathcal{B}$, and the structure varies smoothly in the obvious sense. It is important, however, to note that it does not seem possible to define this stack over the Grothendieck topology of complex manifolds and open covers; in other words, it does not appear possible to discuss chiral CFT's with $D$-branes. To see this, we consider the following

Example: The moduli space of elliptic curves $E$ with an unparametrized hole (i.e. one closed $D$-brane component with a given label). It is easily seen that the moduli space of such worldsheets is the ray $(0, \infty)$, i.e. not a complex manifold. To see this, the key point is to notice that the invariant $\operatorname{Im}(\tau)$ of the elliptic curve $F$ obtained by attaching a unit disk to $E$ along the $D$-brane component does not depend on its parametrization. Intuitively, this seems plausible since $\operatorname{Im}(\tau)$ is the "volume". To rigorize the argument, we first recall that if one cuts the elliptic curve along a non-separating curve, then $\operatorname{Im}(\tau)$ can be characterized as the "thickness" of the resulting annulus (every annulus is conformally equivalent to a unique annulus of the form $S^{1} \times[0, r]$ for some boundary parametrization; $r$ is the thickness). But now any reparametrization of the $D$-brane component $c$ of $E$ is a composition of reparametrizations which are identity outside of a certain small interval $J \subset c$. Thus, it suffices to show that the invariant $\operatorname{Im}(\tau)$ does not change under such reparametrizations. However, we can find a smooth non-separating curve $d \supset J$ in $F$; then cutting $F$ along $d$, the said change of parametrization of $c$ becomes simply a change of parametrization of one of the boundary components of $F$; we already know that does not affect thickness.

By a $K$-labelled closed/open $C F T$ ( $D$-brane category) with 1-dimensional anomaly $\left(H_{i}\right)_{i \in K}$ we shall mean a CFT with 1-dimensional modular functor on the SLCMC $\mathcal{B}$ over the stack of lax monoids $S_{K}$, with target in the SLCMC $\underline{H}_{K}$. This means a lax morphism of SLCMC's

$$
\tilde{\mathcal{B}} \rightarrow\left(\underline{H}_{i}\right)_{i \in K}
$$

where $\tilde{\mathcal{B}}$ is a $\mathbb{C}^{\times}$-central extension of $\mathcal{B}$. These concepts were defined in [14] (see the Appendix for a review).

From this point of view, we see that the question of classifying $D$-branes for a given (closed) CFT $H$ is, at least a priori, not well stated: it is possible that there may be different $D$-brane categories on the same set of objects, or even with the same endomorphism monoids (the state spaces $H_{a a}$ ), but with different choices of $H_{a b}$. One may therefore ask for a classification of all possible $D$-brane categories for a given closed CFT.

We shall, however, not follow that route in this Section. Instead, we would like to capture the more basic physical intuition that $D$-branes should be objects with their own properties, which determine in some canonical way the morphisms 
between them. Therefore, we shall restrict our attention, instead of classifying all possible $D$-brane categories, to attempting to build one canonical $D$-brane category.

For a hint of what such category would have to look like, we follow the approach of Moore-Segal 22. They suggested that, in the case of 2-dimensional topological quantum field theory (TQFT), $D$-branes should be, at least under some assumptions, (finitely generated projective) modules over the algebra $A$ associated with the TQFT. Therefore, they reach the conclusion that $D$-branes in TQFT are classified by $K^{0}(A)$, although of course more precisely $K^{0}(A)$ is the Grothendieck group of the category of $D$-branes and isomorphisms.

It is very appealing to try to find an analogue of the same treatment for $D$ branes in conformal field theory. In particular, one can ask what is the right notion of "module" over a CFT which would correspond to $D$-branes. It is important to note that the right notion does not seem to be the CFT analogue of modules over a VOA, which play an important role in the investigation of chiral CFT's (since they determine their modular functor). However, the $D$-brane classification question can be asked even for CFT's with 1-dimensional anomaly, and, as we already saw, $D$-branes on the other hand cannot be chiral. While we don't think the analogues of VOA modules for non-chiral theories have been investigated, the analogy with the chiral case suggests that one would see only one module over any CFT with 1-dimensional anomaly, and that, in any case, this notion of module seems to be the wrong one.

We shall next propose what seems like a simplest possible notion of " $D$-brane module". Therefore, we also construct an " $D$-brane cohomology" which is the $K$ theory of this category of $D$-brane modules. While we do not investigate tensor products in this paper explicitly, it should however be noted that our $D$-brane cohomology is a module over ordinary $K$-theory in the sense of algebraic topology; this means that $D$-brane cohomology should be, in itself, considered a kind of $K$ theory; it does not appear to contain the type of homotopy-theoretical information which we would expect, for example, in elliptic cohomology. It also does not appear to have the type of modularity associated with elliptic cohomology.

Our notion of $D$-brane module captures the simplest possible feature required of open string theory - propagation along a single $D$-brane, with built in correlation with closed CFT events. Mathematically, this is done as follows.

To define a $D$-brane module, we must introduce a certain special partial SLCMC $\mathcal{P}$. Namely, we will consider two labels, 1 (closed) and $m$ (open, or "module"). We will consider open string worldsheets $X$.

However, we shall impose the following special condition: The $D$-brane components of $X$ are unlabelled. In addition, in each connected component of the worldsheet, there are no closed $D$-brane components, and there are either no $D$ brane comoponents at all, or alternately precisely two open string parametrization components $\alpha$ and $\omega$, which lie on the same boundary component, and moreover $\alpha$ is oriented inbound and $\omega$ outbound. We let these be the LCMC forming the sections over a point of the partial SLCMC $\mathcal{P}$; (it is extended to an SLCMC in the canonical way described in [14. 
Note that $\mathcal{P}$ is "almost an SLCMC": the only reason it is partial is that we do not allow to glue the inbound and outbound open string component of the same connected component together, which would produce two closed $D$-brane components, which we do not allow.

Now a $D$-brane module $H_{a}$ over a closed CFT $H$ is defined to be an abstract CFT with 1-dimensional anomaly over the partial SLCMC $\mathcal{P}$, where the Hilbert space assigned to label 1 , resp. $m$ is $H$, resp. $H_{a}$. Note that it makes sense to define a sub- $D$-brane module, and that, of course, a set-theoretical intersection of an arbitrary set of $D$-brane modules is again an $D$-brane module. We can, therefore, speak of an $D$-brane module $H_{a}$ spanned by a set of elements of $H_{a}$. We say that an $D$-brane module is finitely generated if it is spanned by a finite set of elements. Note, also, that isomorphism of $D$-brane modules is well defined in the obvious way (due to the fact that the SLCMC's of Hilbert spaces are "strict", in the sense that the only laxness comes from the underlying lax-commutative monoid of sets). It is slightly less obvious that $D$-brane modules with the same 1-dimensional anomaly also have a well defined direct sum: to get the sum of $H_{a}$ and $H_{b}$, the Hilbert space attached to the label 1 remains $H$, but the Hilbert space attached to $m$ is $H_{a} \oplus H_{b}$. The point is that the vacuum operator on each connected worldsheet in $\mathcal{P}$ can be interpreted as an element of

$$
\bigotimes H \otimes H o m\left(H_{a}, H_{a}\right) \text {. }
$$

Here Hom stands, for example, for bounded homomorphisms. There is, of course, an additional condition that the element (1) be trace class, but it is nevetheless clear that endomorphisms of two Hilbert spaces can be added and that the sum of trace class endomorphisms is trace class. It is then easily verified that such choice of elements extends to a full structure of an $D$-brane module.

Fixing 1-dimensional anomaly $\alpha\left(\mathbb{C}^{\times}\right.$-central extension $)$on $P$, we now define the $D$-brane isomorphism category $C_{\alpha}(H)$ as follows: the objects are finitely generated $D$-brane modules $H_{a}$, and morphisms are isomorphisms of $D$-brane modules. This is a symmetric monoidal category with respect to the direct sum just introduced. Its classifying space is therefore an $E_{\infty}$-space, and the corresponding generalized cohomology theory $D(H)$ is what we may call $D$-brane cohomology, "classifying" stable $D$-brane modules in a manner analogous to Moore-Segal's theorem for 2dimensional TQFT (22).

It is appropriate to comment on how one would approach the construction of a state space of string stretched between two $D$-brane modules, i.e. how to use $D$ brane modules to get an actual $D$-brane category. We remark, however, that we do not have that question solved mathematically. The problem is mainly the topology, or Hilbert space structure: in closed CFT, Hilbert space structure comes from a combination of a symmetric bilinear form got by a thin annulus with two inbound (or outbound) boundary components, and real structure (see 14 for a discussion). Similar ingredients can be used to give a Hilbert structure for the state space $H_{a a}$ (which we are yet to construct). However, no such natural Hilbert structure seems to be present on $H_{a b}$ (just as there isn't on the non-unit labels of RCFT), because these modules are not necessarily contragredient to themselves. 
Nevertheless, the outline the construction of $H_{a b}$ is as follows: consider all possible disk worldsheets $Q$ with two inbound parametrization components and one outbound parametrization component. One of the inbound parametrization components is labelled $a$ and the other one $b$. The other inbound parametrization component is labelled $a b$, and its inbound point abuts the outbound point of the parametrization component labelled $a$, while its outbound point abuts the outbound point of the outbound parametrization component. Now an element of $H_{a b}$ is a collection of trace class elements

$$
f_{Q} \in H_{a}^{*} \hat{\otimes} H_{b}
$$

one for each worldsheet $Q$ as above. The axiom is that whenever any number of the worldsheets $Q$ are glued to any number of worldsheets in $\mathcal{P}$ (preserving labels) and trace of vacua taken (with $f_{Q}$ taken as vacuum on $Q$ ), then the resulting element coincides (via the canonical isomorphisms supplied by SLCMC) with the element obtained by similar (but different) gluing which produces an isomorphic worldsheet. It can be shown that state spaces constructed in such way ("relative homomorphisms") do indeed obey axioms of open string CFT in some weak sense, but additional work is needed to explain topology, and the trace class condition.

Finally, we remark that at least in one situation, there is a good candidate at least for the Hilbert space $H_{a a}$. This occurs when $H_{a}$ obeys the $D$-brane category axioms already, with open label set $K^{\prime}=\{a\}$. Note that this is not such an unreasonable assumption, since the SLCMC testing these conditions contains the partial SLCMC $\mathcal{P}$, and there might be a general reason why the stronger condition should occur (see examples below). In that case, we call $a$ an elementary D-brane, and we can set

$$
H_{a a}:=H_{a} .
$$

Of course, it is not guaranteed that this choice will agree with the one made in the previous paragraph (if it does, we call it a simple D-brane). However, note that even starting from simple $D$-branes $a$, we easily obtain $D$-branes which are not elementary: simply take the direct sum $n a$ of $n$ copies of $a$.

The formalism of SLCMC's is very powerful, much more so than operads. However, in order to provide a more concrete taste of our present construction, we note that if we restrict attention to genus 0 worldsheets with one outbound closed boundary component and no open string component or no closed string outbound boundary component per connected component, we can describe the type of module structure we are considering in terms of operads. Concretely, let $\mathcal{C}$ be the operad of connected closed string genus 0 worldsheets with one outbound boundary component. Now consider the space of all connected worldsheets in $\mathcal{P}$ of genus 0 and with no outbound closed string boundary component. Recall that according to the definition of $\mathcal{P}$, in addition to the closed parametrization components, there is one additional boundary component containing two open parametrization components, one inbound and one outbounds. Let $\mathcal{D}(n)$ be the space of all such worldsheets with precisely $n$ inbound closed string boundary components. We may ask what algebraic structure is present on the space $\mathcal{D}$. 
The answer is that there are two structures which commute in the appropriate sense: one is a right $\mathcal{C}$-algebra structure

$$
\mathcal{D}(n) \times \mathcal{C}\left(k_{1}\right) \times \ldots \times \mathcal{C}\left(k_{n}\right) \rightarrow \mathcal{D}\left(k_{1}+\ldots+k_{n}\right) .
$$

The other structure, however, is that of a monoid

$$
\mathcal{D}(m) \times \mathcal{D}(n) \rightarrow \mathcal{D}(m+n) .
$$

It is, of course, possible to describe explicitly how the structures (2), (3) should commute, but it is more convenient to express that in terms of monads: If $C$ is the monad associated with the operad $\mathcal{C}$, then the same construction applied to $\mathcal{D}$ gives a functor $D$ with is a right $C$-functor from spaces into topological monoids (recall that right $C$-function means that we have a natural transformation $D C \rightarrow D$ satisfying the obvious axioms).

Every time we have such $\mathcal{D}$, which we might call right monoid $\mathcal{C}$-algebra, and a $\mathcal{C}$-algebra $R$, we get a notion of an $R$-module with respect to $\mathcal{C}, \mathcal{D}$. The standard example of $\mathcal{D}$ is

$$
\mathcal{D}(n)=\mathcal{C}(n+1),
$$

in which case we obtain what is known as $R$-modules via $\mathcal{C}$ (or $R, \mathcal{C}$-modules). However, the example of $\mathcal{D}$ given above shows there are important examples which do not arise by way of (4). It is interesting that such discussion doesn't seem to have been previously made in the literature.

Now if we restrict the structure $D$-brane module $H_{a}$ to the very special type of genus 0 worldsheets just discussed, we can simply say that $H_{a}$ is a module (in the Hilbert sense) of $H$ with respect to $\mathcal{C}, \mathcal{D}$.

\section{Conformal FiEld theOry AND STRING THEORY}

We will now consider the relationship between conformal field theory and string theory, and the way it reflects on our investigation. As a standing reference on string concepts, we recommend one of the standard textbooks on the subject, e.g. 12 or 26. One added feature of string theory is, of course, supersymmetry, but we shall see soon that this turns out not to be the only complication.

We will, therefore, begin our discussion with bosonic string theory (superstrings will enter later). The essential point of string quantization is that conformal field theory quantizes parametrized strings, while physical strings should be unparametrized. Now to pass from parametrized strings to unparametrized, one needs a way to quantize the complex structure. This problem is analogous to gauge fixing in gauge theory. In fact, this is more than just an analogy: from a strictly worldsheet point of view, conformal field theory is indeed a 2-dimensional quantum field theory satisfying Schwinger axioms, and can be viewed as a gauge theory in a certain sense; however, we do not need to pursue this here. The important point is that in string theory, complex structure gauge is in fact needed to produce a consistent theory: conformal field theory is anomalous and, in Minkowski space, contains states of negative norm.

The modern approach to gauge fixing in gauge theory, and to string quantization, is through Fadeev-Popov ghosts and BRST cohomology. In the string theory case, 
we start with a CFT $H_{m}$, the (matter $C F T$ ). In this case, BRST cohomology is essentially a semi-infinite version of Lie algebra cohomology of the complexified Witt algebra (viewed as a "Lie algebra of the semigroup of annuli") with coefficients in $H_{m}$. To be precise about this, we must describe the semiinfinite analogue of the complex $\Lambda(g)$ for a Lie algebra $g$ where $g$ is the Witt algebra. As it turns out, this semiinfinite Lie complex is also a CFT which is denoted as $H_{g h}$ and called the Fadeev-Popov ghost CFT. A mathematical description is outlined in 29, and given in more detail in [16. In the chiral CFT case, $H_{g h}$ is a Hilbert completion (with a chose Hilbert structure) of

$$
\Lambda\left(b_{n} \mid n<0\right) \otimes \Lambda\left(c_{n}, n \leq 0\right) .
$$

In the physical case, both chiralities are present, and $H_{g h}$ is a Hilbert completion of the tensor product of (5) with its complex conjugate. To understand why this is a semiinfinite Lie complex of the Witt algebra, we write the generators of (51) as

$$
\begin{aligned}
& b_{n}=z^{-n+1} \frac{d}{d z} \\
& c_{n}=z^{-n-2}(d z)^{2} .
\end{aligned}
$$

So, the $b_{n}$ 's are vector fields on $S^{1}$ (elements of the Witt algebra) and the $c_{n}$ 's are dual to the $b_{-n}$ 's. An ordinary Lie complex would be the exterior algebra on the duals $c_{n}, n \in \mathbb{Z}$. However, a special feature of CFT is a choice of vacuum which allows us, even before gauge fixing, to define correlation functions which are finite, albeit anomalous: this is the mathematical structure known as Segal-type CFT, which we have axiomatized in [14 and here. However, this choice of vacuum of $H_{m}$ is what prompts the "semininfinite approach", where the exterior generators of $H_{g h}$ are not all of the $c_{n}$ 's, but half of the $c_{n}$ 's and half of the $b_{n}$ 's, as in (5). Now it turns out that for our further discussion, it will be important to know explicitly one part of the Virasoro action on $H_{g h}$, namely the conformal weights, or eigenvectors of $L_{0}$. One may guess that $b_{n}, c_{n}$ should be eigenvectors of conformal weight $-n$, but it turns out that one must decrease the conformal weights of the entire complex (5) by 1 , so the correct conformal weight of a monomial in the $b_{n}$ 's and $c_{n}$ 's is $-k-1$ where $k$ is the sum of the subscripts of the $b_{n}$ 's and $c_{n}$ 's in the monomial. In fact, it turns out that the vacuum of the ghost theory, i.e. the element of $H_{g h}$ assigned to the unit disk, is

$$
b_{-1} c_{0} .
$$

Now the ghost CFT has an anomaly which is described by a 1-dimensional modular functor $L$ which has central charge -26 in the chiral case (see [29, [16]) and $(-26,-26)$ in the physical case. (In the chiral case, there is an additional complication that $L$ must be considered a super-modular functor, see [16] and Section 5 below.) A CFT $H_{m}$ is called critical if it has anomaly described by the 1-dimensional modular functor $L^{\otimes-1}$. The 26 'th power of the 1-dimensional free bosonic CFT described (briefly) in the next section is critical in the physical sense (with both chiralities).

Now for a critical CFT $H_{m}$, there is a certain differential $Q$ (called BRST differential) on the (non-anomalous) CFT

$$
\mathbb{H}=H_{m} \hat{\otimes} H_{g h} .
$$


In the chiral case, one has explicitly

$$
Q=\sum_{r \in \mathbb{Z}} L_{r}^{H_{m}} c_{-r}-\frac{1}{2} \sum_{r, s \in \mathbb{Z}}(r-s): c_{-r} c_{-s} b_{r+s}:-c_{0}
$$

(see [5], formula (4.59)). Here $L_{r}^{m}$ are the Virasoro generators acting on $H_{m}$, and $c_{n}, b_{n}, n \in \mathbb{Z}$ are now understood as operators on $H_{g h}$ in the standard way (see [5]). In the non-chiral case, one must add to (8) its complex conjugate. $Q$ is a differential, which means that

$$
Q Q=0 .
$$

The cohomological dimension is called the ghost number. The $c_{n}$ 's have ghost number 1 , the $b_{n}$ have ghost number -1 , so the ghost number degree of $Q$ is +1 . We shall fix the ghost number as an algebra grading, so 1 has ghost number 0 , but other conventions also exist.

What is even more interesting than (10), however, is that $Q$ turns $\mathbb{H}$ into a "differential graded CFT". If we use the usual notation where we write for a CFT, as a lax morphism of SLCMC's,

$$
X \mapsto U_{X},
$$

then we may define a differential graded CFT by the relation

$$
\sum(1 \otimes \ldots \otimes Q \otimes \ldots 1) U_{X}=0,
$$

with the correct sign convention. For simplicity, we assumed in (11) that all boundary components of $X$ are outbound, the adjoint operator $Q^{*}$ is used on inbound boundary components. Physically, (11) is due to the fact that $Q$ is a conserved charge corresponding to the Noether current of a supersymmetry (called BRST symmetry) of the Lagrangian of $\mathbb{H}$ (see [5]).

In any case, we now see that the BRST cohomology

$$
H=H^{*}(\mathbb{H}, Q)
$$

is a non-anomalous CFT. Infinitesimally, this implies that

$$
\left[Q, L_{n}\right]=0
$$

where $L_{n}$ are the standard Virasoro algebra (in our case in fact Witt algebra) generators. However, more is true. In fact, one has

$$
L_{n}=\left[Q, b_{n}\right]
$$

so

$$
Q x=0 \Rightarrow L_{n} x \in \operatorname{Im}(Q) .
$$

Because of (13), $L_{n}$ actually act trivially on $H$, so $H$ is in fact a TQFT (which means that $U_{X}$ only depends on the topological type of $X$ ). Therefore, our machinery would certainly seem to apply to $H$, in fact so would that of Moore-Segal 22. There are, however, two difficulties.

First of all, $H_{m}$ may not actually be a CFT as we defined it because of convergence problems. For example, when $H_{m}$ is the free bosonic CFT on the $(25,1)$ dimensional Minkowski space, the inner product on the space $H_{m}$ is indefinite, so this space cannot be Hilbert-completed with respect to its inner product. This is 
more than a technical difficulty: in physical language, this is the cause of the 1loop divergence of bosonic string theory. In our language, this means that the state space of our would-be TQFT is infinite-dimensional, so $U_{E}$ for an elliptic curve $E$ is infinity, or more precisely undefined. So there isn't, in fact, any variant of the $(25,1)$-dimensional free bosonic CFT for which the machinery outlined above would work mathematically and produce a true TQFT.

In physics, this is an argument why the free bosonic string theory is not physical, and one must consider superstring theory. Our definition of CFT works on the SLCMC of superconformal surfaces, but the convergence problems persist, i.e. again, for the free $(9,1)$-dimensional super-CFT, the BRST cohomology would be TQFT is infinite-dimensional. Physicists argue that the (infinite) even and odd parts of the TQFT are "of equal dimension", and thus the 1-loop amplitude vanishes (a part of the "non-renormalization theorem"). However, we do not know how to make this precise mathematically.

There is another, more interesting caveat, namely that $H$ is actually not exactly the object one wants to consider as the physical spectrum of string theory. Working, for simplicity, in the bosonic case, one usually restricts to states of ghost number 0 , which, at least in the free case, is isomorphic to the quotient $H_{0}$ of the submodule $Z_{0} \subset H_{m}$ of states $x \in H_{m}$ satisfying $L_{n} x=0$ for $n>0$ and $L_{0} x=x$, by the submodule $B_{0}$ of states of null norm (the Goddard-Thorn no ghost theorem). In bosonic string theory, the vacuum of $H_{m}$ is in $H_{0}$, but this is a tachyon, which is not the vacuum of $H$ : the vacuum in $H$ is $b_{-1}$, as remarked above, and has ghost number -1 . In superstring theory, the tachyon is factored out by so called GSO projection, while the vacuum of course persists, and has also ghost number -1 , but a different name, due to the different structure of the theory, which we have no time to discuss here.

One may ask what mathematical structure there is on $H_{0}$ itself. Here the answer depends strongly on whether we work chirally or not. In the chiral case, Borcherds [4] noticed that $H_{0}$ is a Lie algebra. The Lie algebra structure comes from $[u, v]=u_{0} v$ where $u_{0}$ is the residue of the vertex operator $Y(u, z)$. The Jacobi identity follows immediately from vertex operator algebra Jacobi identity. From CFT point of view, this operation is analogous to the Lie bracket in BatalinVilikovisky algebras.

However, when both chiralities are present (which is the case we are interested in), the rabbit hole goes deeper than that. First note that the CFT vertex operator is not holomorphic, and curve integrals do not seem to be the right operations to consider. Instead, elements of $Z_{0}$ are operator-valued $(1,1)$-forms, and therefore can be naturally integrated over worldsheets. Indeed, one can see that integration of an element of $Z_{0}$ over worldsheets produces an infinitesimal deformation of CFT. Elements of $B_{0}$ also deform the CFT, but only by a gauge transformation, so elements of $H_{0}$ give rise to infinitesimal deformations of string theory. We may therefore (despite potentially serious convergence problems) wish to consider a moduli space $M$ of string theories, to which $H_{0}$ is a tangent space at one point. In fact, points of the curved space $M$ should be the true states of string theory, while the points of the tangent space $H_{0}$ are only an approximation. In the physical 
theory, one conjectures that the space $M$ contains all of the 5 original superstring theories as states, and a continuum of states in between. As seen even by studying the basic example of toroidal spacetime, some states in $M$ differ only by "boundary conditions on open strings", and such conditions are called $D$-branes. When there is a well defined spacetime manifold $X, D$-branes as a rule are associated with submanifolds of $X$ with some additional structure. These, however, are classical and not quantum objects (cf. Polchinski [26]), so that approach also has its drawbacks. While rigorous mathematical attempts to define and investigate $D$-branes from the manifold point of view have (with some success) also been made in the literature exist, the "tangent" CFT approximation which we consider here is, in some sense, complementary. Finding a mathematical theory which would unify both points of view is an even much more complex task, which we do not undertake here.

\section{An example: The 1-Dimensional free scalar CFT}

We shall now give the standard examples of $D$-branes in the free bosonic CFT in dimension 1 (which is the CFT description of the linear $\sigma$-model). Unfortunately, even for this most basic CFT, a mathematically rigorous description of its convergence issues is nowhere to be found in the literature. The best outline we know of is given in [29].

A good first guess for the free (bosonic) field theory state space is, analogously with the lattice theories (see [14)

$$
H=L^{2}(\mathbb{R}, \mathbb{C}) \hat{\otimes} S \hat{y} m<z^{n}, \bar{z}^{n} \mid n>0>.
$$

Here $L^{2}(\mathbb{R}, \mathbb{C})$ denotes $L^{2}$-functions with respect to the Gaussian measure. The quantum number associated with this space is the momentum.

To be more precise, (14) should be a Heisenberg representation of a certain infinite-dimensional Heisenberg group. To describe it, we start with the topological vector space $V$ of all harmonic functions on $S^{1}$ or, more precisely, harmonic functions on small open sets in $\mathbb{C}$ which contain $S^{1}$ and, say, the topology of uniform convergence in an open set containing $S^{1}$. Thus, $V$ is topologically generated by the functions

$$
z^{n}, \bar{z}^{n}, n \in \mathbb{Z}
$$

and

$$
\ln \|z\| \text {. }
$$

To define the Heisenberg group, we would like to find a $\mathbb{C}^{\times}$-valued cocycle on $V$ which would be invariant under the action of $\operatorname{Diff}^{+}\left(S^{1}\right)$. However, similarly as in the case of lattice theories, we do not know any such cocycle. Instead, one considers the space $U$ of harmonic $\mathbb{C}$-valued functions on (an open domain containing) the unit interval $I$. The point is that the harmonic functions on $I$ break up into holomorphic and antiholomorphic parts; a topological basis of the holomorphic part is given by the elements

$$
z^{n}, n \in \mathbb{Z}, \ln (z),
$$

and a topological basis of the antiholomorphic parts is given by their complex conjugates. Therefore, the holomorphic and antiholomorphic parts $U_{+}$and $U_{-}$ of $U$ have well defined winding numbers which can be added to a total winding 
number; let $V^{\prime} \subset U$ be the set of functions of total winding number 0 . Then the $\operatorname{map} \exp (?)=e^{2 \pi i ?}$ gives a projection

$$
V^{\prime} \rightarrow V
$$

whose kernel consists of the constant functions. Now to get the free field theory, one proceeds analogously to lattice theories (see [14]), specifying a cocycle on $U$. We shall specify separately cocycles on both $U_{+}$and $U_{-}$. However, because the integrality condition is replaced by equality of winding numbers on $U_{+}$and $U_{-}$, we have more freedom in choosing the cocycle. For example, we can put, on both $U_{+}$ and $U_{-}$,

$$
c(f, g)=\exp \frac{1}{2}\left(\int_{S^{1}} f d g-\Delta_{f} g(0)+\frac{1}{2} \Delta_{f} \Delta_{g}\right),
$$

(where $\Delta_{f}$ is the winding number). The effect of this is that if we apply the cocycle to lifts of two harmonic functions $f, g$ on a worldsheet to its universal cover, whose restriction to boundary components are $f_{i}, g_{i}$ (as is done in 14 for the lattice theories), the Greene formula implies that

$$
\begin{aligned}
& c(f, g)=\exp \frac{1}{4}\left(\sum_{i<j}\left(\Delta_{f_{i}} \Delta_{g_{j}}+\Delta_{g_{i}} \Delta_{f_{j}}\right)+\sum_{i=1}^{n} \Delta_{f_{i}} \Delta_{g_{i}}\right)= \\
& \exp \frac{1}{4}\left(\sum_{i=1}^{n} \Delta_{f_{i}} \sum_{i=1}^{n} \Delta_{g_{i}}\right)=1 .
\end{aligned}
$$

Thus, the situation is simpler than in the case of lattice theory. Now similarly as in the case of lattice theory, the cocycle $c$ we have constructed, when restricted to $V^{\prime}$, is trivial on the constant functions, so we get a canonical map

$$
\mathbb{C} \rightarrow \tilde{V}^{\prime}
$$

(where ? denotes Heisenberg group with respect to a given cocycle). Similarly as in the case of lattice theories, in fact, $c(f, g)=0$ for $f, g \in V^{\prime}, f$ constant, so the subgroup (17) is normal, so the desired Heisenberg group can be defined by

$$
\tilde{V}=\tilde{V}^{\prime} / \mathbb{C} .
$$

Then $H$ should be the Heisenberg representation of the right real form of (18). Note, however, that the above construction comes with no obvious natural choice of real form. Let us postpone the discussion of this issue, as we shall see it is related to the convergence issues of the CFT. Now, conformal field theory structure is specified as usual: looking at the Heisenberg representation $H_{\partial X}$ of the central extension $\tilde{V}_{\partial X}$ of the space $V_{\partial X}$ of harmonic functions on the boundary of a worldsheet $X$, we have already constructed a canonical splitting of the pullback of the central extension to the subspace $V_{X}$ of harmonic functions on $X$; we would like to define the field theory operator associated with $X$ as the vector space of invariants of $H_{\partial X}$ with respect to $V_{X}$.

A usual "density argument" (cf. 29, 14, 27]) shows that the invariant vector space $H_{X}$ is always at most 1-dimensional. In more detail, if we denote by $\operatorname{Harm}(X)$ the space of harmonic functions on $X$ and by $\operatorname{Harm}(\partial X)$ the space of 
harmonic functions on a small neighborhood of $\partial X$, and also by $D$ the unit disk, then, by restriction, we may form the double coset space

$$
\operatorname{Harm}(X) \backslash \operatorname{Harm}(\partial X) / \prod_{\partial} \operatorname{Harm}(D)
$$

(the product is over boundary components of $X$ ). Then (19) is isomorphic to $H^{1}(\bar{X}, \underline{\text { Harm }}) \cong \mathbb{C}$ where $\underline{\text { Harm }}$ is the sheaf of harmonic functions and $\bar{X}$ is the worldsheet obtained from $X$ by gluing unit disks on the boundary components. Since $H$ can be interpreted as a (completed) space of functions on

$$
\operatorname{Harm}(\partial X) / \prod_{\partial} \operatorname{Harm}(D),
$$

the identification of (19) shows that only functions on the orbits $\mathbb{C}$ have a chance to be $\operatorname{Harm}(X)$-fixed points. However, studying further the constant functions in $\operatorname{Harm}(X)$, we see that only functions supported on $\{0\} \subset \mathbb{C}$ have a chance of being fixed points.

These observations also point to a difficulty with a Hilbert space model for $H$. What kind of reasonable Hilbert space functions on $\mathbb{R}$ contain distributions supported on a single point? Now recall the reason why the Hilbert space is not yet fixed: we haven't fixed the real structure on $V$. One clue for such real structure is that, from the desired interpretation of $H$ as functions on (20),

$$
A=\operatorname{Harm}(D) \subset V
$$

should be our "Lagrangian subspace" so that $H=\widehat{S y m(A)}$, (cf. [27], Section 9.5). Thus, we can define the real structure on $V$ by specifying the inner product on $A$. The choice enjoying the desired invariances is

$$
\langle f, g\rangle=\int \bar{f} d g .
$$

But then this is only a semidefinite Hermitian product on $A$, where constants have norm 0 ! The above discussion shows that this is more than a technical difficulty. To get the field operator $U_{X}$ to converge, we must take the inner product (21), which leads to

$$
H=\prod_{k \in \mathbb{R}} \operatorname{Sym}\left\langle\widehat{z^{n}, \bar{z}^{n} \mid} n>0\right\rangle
$$

( $\prod$ is the Cartesian product). Physically, the quantum number $k$ is the momentum. Then the notion of "Hilbert product" of copies of $H$ and "trace" must be adjusted. For more details, see the Appendix. With these choices, convergence of $U_{X}$ can be proven similarly as in 14] for lattice theories (e.g. using boson-fermion correspondence at 0 momentum), so the free bosonic CFT is rigorous. This convergence problem does not arise if we consider the $\sigma$-model on a compact torus instead of flat Euclidean space.

For completeness, we note that we haven't discussed inbound boundary components, and closed worldsheets. The former topic offers no new phenomena and can be treated simply by reversing the sign of the cocycle. Discussing closed worldsheets amounts really to discussing in detail the anomaly, which is $H^{1}(X, \underline{\operatorname{Harm}})$, analogously to the fermionic case treated in [16]. 
Now we want to give examples of simple elementary $D$-branes in the free CFT. The above discussion shows that we would have to work in compact spacetime (a torus) to make the examples fit the scheme proposed in Section 2 literally. However, we elect instead to stick to flat spacetime $\mathbb{R}$, where the situation seems more fundamental. It must be then understood, however, that the notion of $D$-brane module in this situation must also be generalized in a way analogous to CFT (as discussed in the Appendix), to solve the convergence issue.

Consider the $1 / 2$-disk $B$ consisting of elements of $D$ with non-negative imaginary part. We consider $B$ an open string worldsheet where the real boundary elements are the $D$-brane component, and the open string component is parametrized by the map $e^{\pi i t}$. Then we can consider the space of all harmonic functions on the boundary of $B$ which obey a suitable boundary condition on the $D$-brane component. The boundary conditions allowable first of all must be conformally invariant. The most obvious such condition is that the derivative of the function in question be 0 in the direction of a certain vector $u \in S^{1}, \operatorname{Im}(u)>0$ or $u=1$. A priori, all of those conditions are allowable. However, if we want to follow the methods we used above to describe closed free CFT, additional conditions are needed. Namely, we need the vector space of functions satisfying the condition to have a central extension which is a Heisenberg group. Moreover, to get consistency of open and closed CFT, we need the Heisenberg group to be obtained by restriction of the cocycle (15). This means that the cocycle (15) or more precisely the bilinear form by whose exponentiation the cocycle arises, must be non-degenerate on the vector space of functions we are allowing. This happens for the case $u=i$, although in order for it to work, we must allow the harmonic function $\ln \|z\|$, which has a logarithmic singularity on the $D$-brane boundary component of $B$ ! Nevertheless, this case works, and gives the classical free open string theory (von Neumann boundary condition). The corresponding space of functions for $u=1$ is polarized canonically (by the subspace of harmonic functions which extend harmonically to $B$, with possibly logarithmic singularities on the $D$-brane component), so we can take again the bosonic Fock space. This means that we restrict the cocycle (15) to the vector space of harmonic functions obeying the specified boundary condition, and take the Heisenberg representation. The field operator is constructed in the same way as in the closed string case. However, this time it is not immediately obvious that the space $H_{\partial X}$ for an open worldsheet $X$ is isomorphic to the suitably completed tensor product of the state spaces of the individual parametrization components. Nevertheless, this can be proven by sewing on copies of the half-disk $B^{-}$with opposite parametrization; the resulting gluing map proves that the space $H_{\partial X}$ is dual to the Hilbert tensor product of the individual copies of $H_{B^{-}}$, as needed.

We have therefore constructed classical open string theory, i.e. the 1-dimensional $D$-brane, but what about the 0 -dimensional $D$-brane, i.e. the open free CFT with a Dirichlet condition? (Note: in physics, one spacetime dimension is time, which is usually not counted in the dimension of the $D$-brane; thus, instead of 1 - and 0 -dimensional $D$-branes, we would speak of $D 0$-brane and $D(-1)$-brane or instanton.) In any case, in the language considered above, $u=i$ was the Von Neumann condition, so $u=1$ should be the Dirichlet condition. However, in the case of $u=1$ we are facing the same difficulties we had for general $u$, namely that the functions satisfying the boundary condition do not extend to a Heisenberg group using the 
cocycle (15). In order to get a Heisenberg group, we must find a way to "get rid of the constant functions", and do so naturally enough to support a field theory. It is not clear how to do that for general $u$, but for $u=1$ there is a special construction which eliminates the constants canonically: take

$$
V_{0}=<f-\bar{f} \mid f \text { is a harmonic function on } \partial B>\text {. }
$$

It can be shown that, using the cocycle (15), the Heisenberg representation $H_{0}$ of $\tilde{V}_{0}$ is indeed an open string theory together with the free closed string theory $H$. We see that the space $H_{0}$ misses the momentum quantum number, but this doesn't mean the momentum is constant; rather, as it turns out, the appropriate interpretation is that the position is constant, and the space $H_{0}$ is the state space of a $D$-brane of the free field theory $H$. We get a different elementary $D$-brane for each choice of position.

We may ask if the $D$-branes at different positions we just described are isomorphic as open CFT's of the 1-dimensional closed free bosonic CFT. The answer is that they are isomorphic, but not over the identity automorphism of the closed CFT. The free bosonic CFT has automorphisms coming from translations: $D$ branes of positions $\mu, \lambda$ are isomorphic over the closed CFT automorphism given by translation by $\lambda-\mu$.

\section{CFT ANOMALY VIA 2-VECTOR SPACES AND ELLIPTIC COHOMOLOGY}

In this section, we give a new definition of modular functor which generalizes the definition given in 14]. Consider a free finitely generated lax module $\mathcal{M}$ over the lax semiring $\mathbb{C}_{2}$ (the category of finite-dimensional $\mathbb{C}$-vector spaces; it is convenient to let the morphisms of $\mathbb{C}_{2}$ be all linear maps; thereby, $\mathbb{C}_{2}$ is not a groupoid, and we have to use the version of lax algebra theory which works over categories - see the appendix; of course, it is possible to consider the subcategory $\mathbb{C}_{2}^{\times}$of $\mathbb{C}_{2}$ whose morphisms are isomorphisms). Consider further $\mathbb{C}_{2}^{\text {Hilb }}$, the $\mathbb{C}_{2}$-algebra of Hilbert spaces with the Hilbert tensor product. We put $\mathcal{M}^{\text {Hilb }}=\mathcal{M} \otimes_{\mathbb{C}_{2}} \mathbb{C}_{2}^{\text {Hilb }}$. Now consider $H \in \in_{1} \mathcal{M}^{H i l b}$ (the symbol $\in_{1}$ means a map of lax $\mathbb{C}_{2}$-modules $\mathbb{C}_{2} \rightarrow$ ?; in our case, this is the same thing as $\left.H \in \operatorname{Obj}\left(\mathcal{M}^{H i l b}\right)\right)$. We shall define two LCMC's $C(\mathcal{M})$ and $C(\mathcal{M}, H)$ (underlying LCM of sets) which are, in standard ways, extended into SLCMC's over the Grothendieck category of finite-dimensional smooth complex manifolds.

The LCMC's are constructed as follows: the objects of $C(\mathcal{M})$ over the pair of finite sets $(S, T)$ are 1-elements of $\mathcal{M}^{\otimes S} \otimes \mathcal{M}^{* \otimes T}$; the morphisms are 2-isomorphisms. Here $\mathcal{M}^{*}$ is the dual lax $\mathbb{C}_{2}$-module of $\mathcal{M}$, whose objects are (lax) morphisms of lax $\mathbb{C}_{2}$-modules $\mathcal{M} \rightarrow \mathbb{C}_{2}$ and morphisms are natural isomorphisms compatible with the operations. The gluing maps are given by trace over $\mathbb{C}_{2}$, i.e. the evaluation morphism $\mathcal{M} \otimes \mathcal{M}^{*} \rightarrow \mathbb{C}_{2}$. An object of $C(\mathcal{M}, H)$ over $(S, T)$ consists of an object $M$ of $C(\mathcal{M})$, and 2-morphism $u: M \rightarrow_{2} H^{\hat{\otimes} S} \hat{\otimes} H^{* \hat{\otimes} T}$ whose image consists of trace class elements: (Choosing a basis of $\mathcal{M}$, a 1-element of $\mathcal{M}^{\text {Hilb }}$ becomes a collection of Hilbert spaces, so $\hat{\otimes}$-powers of $H$ are collections of $\hat{\otimes}$-powers; an element is trace class if each of its components is trace class. For generalizations beyond the trace class context, see Remarks in the Appendix.) Here $H^{*} \in{ }_{1} \mathcal{M}^{*}$ Hilb is defined by 
putting, for $V \in_{1} \mathcal{M}, H^{*}(V)=\operatorname{Hom}_{\mathcal{M}^{H i l b}}(H, V)$. Morphisms are commutative diagrams of the obvious sort. To define gluing operations, note that

$$
u: M \rightarrow_{2} H^{\otimes(S+U)} \hat{\otimes} H^{* \hat{\otimes}(T+U)}
$$

induces a 2-morphism $\operatorname{tr}(u):\left(1 \otimes t r_{1}\right) M \rightarrow_{2} H^{\hat{\otimes} S} \hat{\otimes} H^{* \hat{\otimes} T}$ where $t r_{1}: \mathcal{M}^{\otimes U} \otimes$ $\mathcal{M}^{* \otimes U} \rightarrow \mathbb{C}_{2}$ is the evaluation morphism; it is defined by using the canonical morphism $t r_{2}: t r_{1}\left(H \otimes H^{*}\right) \mathbb{C}$.

To define the corresponding SLCMC's, (which we denote by the same symbols), as usual, it suffices to define sections over pairs of constant covering spaces $(U \times$ $S, U \times T)$ of a complex manifold $U$. For defining $C(\mathcal{M})$, we need a concept of a holomorphically varying 1 -element of $\mathcal{M}$. To this end, we denote by $\operatorname{Hol}\left(U, \mathbb{C}_{2}\right)$ the lax commutative monoid of finite-dimensional holomorphic bundles on $U$. Then the concept we need is

$$
M_{U} \in_{1} \mathcal{M}_{U}:=\mathcal{M} \otimes_{\mathbb{C}_{2}} \operatorname{Hol}\left(U, \mathbb{C}_{2}\right) .
$$

This determines the SLCMC $C(\mathcal{M})$. To define $C(\mathcal{M}, H)$, note that $H$ does not depend on $U$, so in the case of constant covering space described above, we simply need a

$$
u_{U}: M_{U} \rightarrow_{2} H_{U}^{\hat{\otimes} S} \hat{\otimes} H_{U}^{* \hat{\otimes} T}
$$

where $H_{U}$ is the constant $H$-bundle on $U$, and the 2-morphism means a morphism of holomorphic bundles.

Definition: A modular functor on an SLCMC $C$ with labels $\mathcal{M}$ is a (lax) morphism of SLCMC's $\phi: C \rightarrow C(\mathcal{M})$. A CFT on $C$ with modular functor on labels $\mathcal{M}$ with state space $H$ is a (lax) morphism of SLCMC's $\Phi: C \rightarrow C(\mathcal{M}, H)$.

Now note that $C\left(\right.$ ?) is a 2 -functor from the 2 -category $\mathbb{C}_{2}-\bmod$ of lax $\mathbb{C}_{2}$-modules (1-morphisms are equivalences of $\mathbb{C}_{2}$-modules, and 2-morphisms are natural isomorphisms compatible with $\mathbb{C}_{2}$-module structure) into the 2-category of SLCMC's. Similarly, $C\left(\right.$ ?, ?) is a 2-functor from the 2-category $\mathbb{C}_{2}-\bmod _{*}$ of pairs $\mathcal{M}, H$ where $\mathcal{M}$ is a $\mathbb{C}_{2}$-module, and $H \in \in_{1} \mathcal{M}^{\text {Hilb }}$. Here 1-morphisms in $\mathbb{C}_{2}-\bmod , \mathbb{C}_{2}-\bmod _{*}$ are equivalences of lax $\mathbb{C}_{2}$-modules, 2-morphisms are natural isomorphisms compatible with $\mathbb{C}_{2}$-module structure. (In $\mathbb{C}_{2}-\bmod _{*}$, 1-morphisms $(\mathcal{M}, H) \rightarrow(\mathcal{N}, K)$ are 1morphisms $\phi: \mathcal{M} \rightarrow \mathcal{N}$ in $\mathbb{C}_{2}-\bmod$ together with a 2-isomorphism $\lambda: \phi(H) \rightarrow K$; a 2-morphism $\phi \rightarrow \psi$ is a 2-morphism in $\mathbb{C}_{2}-\bmod$ which induces an isomorphism $\phi(H) \rightarrow \psi(H)$ commuting with the $\lambda$ 's.)

We use this to build a 2-category $\Gamma$ of $C F T$ 's as a "comma 2-category". The objects are tuples $\mathcal{M}, H, \Phi$ where $\Phi$ is a CFT on $C$ with labels $\mathcal{M}$ and state space $H, 1$-morphisms are tuples $\Phi, \Psi, f, \iota$ where $\Phi, \Psi$ are CFT's with labels $\mathcal{M}, \mathcal{N}$ and state spaces $H, K, f$ is a $\mathbb{C}_{2}-\bmod _{*}$ - 1 -morphism $(\mathcal{M}, H) \rightarrow(\mathcal{N}, K)$ and $\iota$ is a natural isomorphism $f\left(M_{X}\right) \rightarrow_{2} N_{X}$ where $M_{X}, N_{X}$ are the 1-elements of $\mathcal{M}$, $\mathcal{N}$ assigned to $X \in O b j \mathcal{C}$ by $\Phi, \Psi$ which commutes with SLCMC structure maps and the $u$ 's assigned by $\Phi, \Psi$ (we have used the notation of sections over a point, but we mean this in the stack sense for sections over any complex manifold $U$ ). 2morphisms $\Phi, \Psi, f, \iota \rightarrow \Phi, \Psi, g, \kappa$ are given by 2 -isomorphisms $f \rightarrow g$ in $\mathbb{C}_{2}-\bmod _{*}$ which commute with $\iota, \kappa$ (hence the $u$ 's). 
Next, we shall show that $\Gamma$ is a symmetric monoidal 2-category. This means that we have a lax 2-functor $\oplus$ with the same coherence 1-isomorphisms as in a symmetric monoidal category, but coherence diagrams commute up to 2-cells; the 2 -cells, in turn, are required to satisfy all commutations valid for the trivial 2-cells of coherence diagrams in an ordinary symmetric monoidal category. Thus, the main point is to construct the 2 -functor $\oplus$. Suppose we have two objects $\mathcal{M}, H, \Phi$ and $\mathcal{N}, K, \Psi$ of $\Gamma$. Then their sum is $\mathcal{M} \oplus \mathcal{N}, H \oplus K, \Phi \oplus \Psi$ : The first component is the direct sum in $\mathbb{C}_{2}-$ mod. $H \oplus K$ is the direct sum induced by that functor on 1-morphisms. The symbol $\Phi \oplus \Psi$, however, has to be defined explicitly. For simplicity, we shall restrict to sections over a point. Then, the data which remains to be defined, for an object $X$ of the source SLCMC, is

$$
M \oplus N,
$$

and

$$
u: M \oplus N \rightarrow_{2}(H \oplus K)^{\otimes S} \otimes(H \oplus K)^{* \otimes T} .
$$

Of this data, for $X$ connected, (22) is, again, the direct sum induced by the direct sum of $\mathbb{C}_{2}$-modules on 1-morphisms, composed with the canonical map

$$
\begin{aligned}
& \mathcal{M}^{\otimes S} \otimes \mathcal{M}^{* \otimes T} \oplus \mathcal{N}^{\otimes S} \otimes \mathcal{N}^{* \otimes T} \\
& \rightarrow(\mathcal{M} \oplus \mathcal{N})^{\otimes S} \otimes(\mathcal{M} \oplus \mathcal{N})^{* \otimes T} .
\end{aligned}
$$

Analogously, (23) is given by the $\oplus$ of $\mathbb{C}_{2}$-modules on 2-morphisms, composed with (24). For $X$ non-connected, note that we are forced to define all data by applying the tensor product to the data on connected components. This definition extends to 1-morphisms and 2-morphisms in a standard way to produce a symmetric monoidal 2-category of CFT's $\Gamma$.

Note that we may not always wish to work with the whole $\Gamma$, but with some symmetric monoidal sub-2-category $\Lambda$; for example, we may take direct $\oplus$-sums of copies of a given CFT.

Now there is an infinite loop space machine for 2-categories: for example, Segal's machine. Segal's machine is supposed to construct an $\mathcal{F}$-space, which is a functor from the category $\mathcal{F}$ of finite sets with base point $*$ and based maps into spaces (alternately, one can think of this as a category of partial maps); it is also required that the functor (called $\mathcal{F}$-space) $B$ be special, which means that the product map from $B(n)$ to the product of copies of $B(1)$ by the maps which send all numbers in $\{1, \ldots, n\}$ except $i$ into the basepoint be an equivalence.

Now to produce a special $\mathcal{F}$-space from a symmetric monoidal category $C$, simply consider the category $C(n)$ which is a category of diagrams, whose objects are tuples $\left(x_{T}\right)$ of objects of $C$ indexed by non-empty subsets of $S$, together with isomorphisms

$$
\bigoplus_{i \in T} x_{\{i\}} \cong x_{T}
$$

Morphisms are commutative diagrams of the obvious kind (see [31]). Now $C($ ?) is a functor from $\mathcal{F}$ into categories, so applying the classifying space gives the requisite $\mathcal{F}$-space. It is special by basic theorems about the homotopy types of classifying spaces. 
However, now note that the same definition (25) in the case of a symmetric monoidal 2-category $C$ gives a 2-category $C(n)$. The only difference is that on 1-morphisms, we do not consider merely diagrams commutative on the nose, but up to 2-cells and 2-morphisms are systems of 2-cells which further commute with the 2-cells thus introduced. With that, however, $C($ ?) becomes a (strict) functor from $\mathcal{F}$ into 2-categories.

So, we are done if we can produce a functorial classifying space construction $B_{2}$ on 2-categories, and show that the $\mathcal{F}$-space $B_{2} C($ ?) is special. The latter is a straightforward exercise which we omit. For the former, however, we remark that to define a classifying space of a 2-category $C$, we can first form the bar construction $B_{1}=B(\operatorname{Mor}(C))$, i.e. the bar construction on 2-morphisms. However, if $C$ is lax, then $B_{1}$ is not a category, but composition is defined with respect to a contractible operad (without permutations). The operad $D$ is as follows: the space $D(n)$ is the standard $(n-1)$-simplex and the composition is given by join:

$$
D(k) \times D\left(n_{1}\right) \times \ldots \times D\left(n_{k}\right) \rightarrow D\left(n_{1}\right) * \ldots * D\left(n_{k}\right)=D\left(n_{1}+\ldots+n_{k}\right) .
$$

Nevertheless, it is well known that such $A_{\infty}$-categories still have a classifying space functor (for example, one can "rectify" them by push-forward change of operads to the one point operad without permutations, which encodes associativity).

Thus, we have produced a symmetric monoidal 2-category of CFT's, and an infinite loop space machine for such case. Therefore, we have an infinite loop space $E$. This is related to the kind of construction used in [14 to give a candidate for an elliptic-like cohomology theory and seems like an improvement in the sense that it gives a model for the additive infinite loop structure (a "free" construction was used in [14).

However, Baas-Dundas-Rognes 2] point out that this kind of construction is naive. The problem is that there are not enough isomorphisms of free lax $\mathbb{C}_{2^{-}}$ modules: they are essentially just permutation matrices composed with diagonal matrices of line bundles. In 2, a solution to this problem is proposed, conjecturally calculating the algebraic $K$-theory of $\mathbb{C}_{2}$. The point is to consider, instead of invertible matrices of finite-dimensional vector spaces, numerically invertible matrices, which means that the corresponding matrix of dimensions of the entry vector spaces is invertible.

Unfortunately, this approach does not seem satisfactory for the purposes of CFT: along with an iso $f: \mathcal{M} \rightarrow \mathcal{N}$, we need to consider also the inverse $\mathcal{M}^{*} \rightarrow \mathcal{N}^{*}$ of the adjoint morphism $\mathcal{N}^{*} \rightarrow \mathcal{M}^{*}$; there is no candidate for such inverse when $f$ is only numerically invertible.

Another clue that something else is needed is the following example of $b c$-systems, whose anomaly, it seems, can only be expressed by considering "modular functors with positive and negative parts".

Example: Consider the chiral $b c$-system of $\Omega^{\alpha}$-forms, $\alpha \in \mathbb{Z}$ (see also Section 3 above). The $b c$-system was first considered mathematically by Segal [29], but the observation that the super-modular functor formalism is needed to capture its properties is due to P. Deligne ([16]). In the case, the state space of the $b c$-system 
is the "fermionic Fock space"

$$
\mathcal{F}_{\alpha}=\hat{\Lambda}\left(H_{+} \oplus \bar{H}_{-}\right)
$$

where $H=<z^{n} d z^{\alpha} \mid n \in \mathbb{Z}>$ and $H_{+}$is, say, the subspace $<z^{n} d z^{\alpha} \mid n \geq 0>$. We select some real form to make this a positive definite Hilbert space (cf. [14], Chapter 2 ). Then the modular functor is 1-dimensional, and is given by the determinant line of $\Omega^{\alpha} X$, the space of holomorphic $\alpha$-forms on a worldsheet $X$. The reason why a super-modular functor is needed here is that we are dealing with Grassmanianns, and signs must be introduced when permuting odd-degree variables for the CFT to be consistent; no such signs, however, occur in CFT's with 1-dimensional anomaly as considered above (see [16] for more details).

Thus, it seems that $\mathbb{C}_{2}$ in our definition of modular functors and CFT's should be replaced by some sort of "group completion" which would involve $\mathbb{Z} / 2$-graded vector spaces. A candidate for such construction is given in the next section, although we will see that this comes at the price of substantially increasing technical difficulty.

\section{The group Completion of $\mathbb{C}_{2}$.}

As argued above, it would be desirable to have a group completion $\hat{\mathbb{C}}_{2}$ of $\mathbb{C}_{2}$ over which we could do the analogues of all of our constructions as suggested by Baas-Dundas-Rognes [2]. In this Section, we propose such construction. However, as also pointed out in [2], any such construction is necessarily accompanied by sustantial difficulties. The first problem is to even define what we mean by "group completion". It is easy to show that for a lax commutative ring $R, B R$ is always an Eilenberg-MacLane space (and hence cannot be used for our purposes), but there is strong evidence that a large class of weaker categorical notions of "weakly group-complete" lax commutative semirings suffer from the same problem [33.

We take an alternate approach of introducing topology into the picture. This means, we construct a model of a topolocial lax commutative semiring $\hat{\mathcal{C}}_{2}$ where there is an object -1 so that $1 \oplus(-1)$ is in the same connected component of 0 . While this approach does seem to lead to a viable definition, one must overcome a variety of technical difficulties caused by the additional topology.

The first issue is what is the appropriate 2-category TCat of topological categories? The point is that requiring functors to be continuous on objects appears to restrict too much the notion of equivalence of topological categories, and consequently alter their lax colimits. To remedy this situation, we define 1-morphisms $\mathcal{C} \rightarrow \mathcal{D}$ in $T$ Cat to be of the form

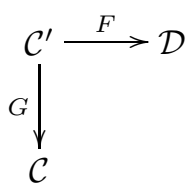

where $F$ is a continuous functor and $G$ is a partition which we define as follows: A partition is given by a topological space $X$ and a continuous map

$$
f: X \rightarrow \operatorname{Obj}(\mathcal{C})
$$


such that the topology on $\operatorname{Obj}(\mathcal{C})$ is induced by $f$ (we work in the category of weakly Hausdorff compactly generated topological spaces). Then we have $\operatorname{Obj}\left(\mathcal{C}^{\prime}\right)=X$, $\operatorname{Mor}\left(\mathcal{C}^{\prime}\right)$ is a pullback of the form

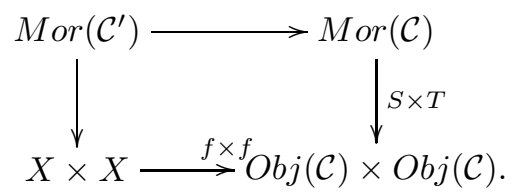

Two functors $F_{1}, F_{2}$ as in (27) are considered equal if they coincide on a common partition, i.e. we have a commutative diagram

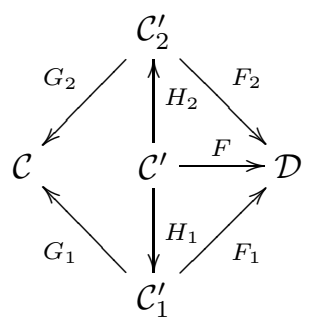

where $H_{1}, H_{2}$ are partitions. Composition is defined by pullback in the usual way, using the following

Lemma 1. If we have a pullback

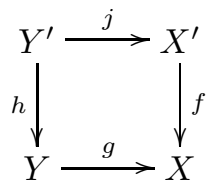

in the category of compactly generated weakly Hausdorff spaces such that $f$ induces the (compactly generated) topology on $X$, then $h$ induces the (compactly generated) topology on $Y$.

Proof: Suppose $V \subset Y, v \in(Y-V) \cap C l(V), h^{-1}(V)$ closed. Then there exists $K$ compact where $v$ is a limit point of $K \cap V$. So, we may replace $V$ by $K \cap V$ and assume $C l(V)=K=Y$. Next, let $Z=g(K)$, so $Z$ is compact.

Case 1: $g(V) \neq Z$. Then there exists $T \subset X^{\prime}$ compact, $T \cap f^{-1}(g(V))$ not closed. Consider the pullback

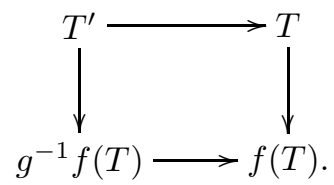

Then $T^{\prime}$ is compact since $g$ is proper. But then $j\left(T^{\prime} \cap h^{-1}(V)\right)=f^{-1}(g(V)) \cap T$, so $T^{\prime} \cap h^{-1}(V)$ cannot be closed in $T^{\prime}$ (since $j \mid T^{\prime}$ is closed, $T^{\prime}$ being compact). This is a contradiction.

Case 2: $g(V)=Z$. Then in particular, there exists $z^{\prime} \in V, g(v)=g\left(z^{\prime}\right)=: z$. Now we may assume that

$v$ is a limit point of $V \cap g^{-1}(\{z\})$. 
Indeed, otherwise, since $K=Y$ is compact weakly Hausdorff, it is normal, hence regular and there exist $U, W$ open in $K, U \cap W=\emptyset, v \in U, C l\left(V \cap g^{-1}(\{z\})\right) \subset W$. But then we may replace $Y$ by $Y-V$ (and $X$ by $g(Y-V)$ ), and we are back to Case 1. So we may assume (29). But then we may replace $X$ by $\{z\}$ and $Y$ by $g^{-1}(\{z\})$. But then (28) is a product, in which case the statement of the Lemma is obviously true (a product projection induces the topology on its target in the compactly generated weakly Hausdorff category). Thus, we have a contradiction again.

Now 2-morphisms in TCat are defined as follows: we can assume we have two 1-morphisms $F_{1}, F_{2}$ given as

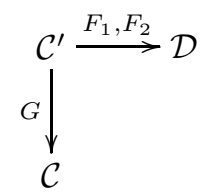

where $G$ is a partition. Then a 2 -morphism is given by a partition

$$
\mathcal{C}^{\prime \prime} \stackrel{G^{\prime}}{\longrightarrow} \mathcal{C}^{\prime}
$$

and a continuous natural transformation

$$
F_{1} G^{\prime} \rightarrow F_{2} G^{\prime} .
$$

Again, two 2-morphisms are identified if they coincide after pullback via a partition, similarly as above.

This completes the definition of the 2-category TCat. This 2-category is defined in such a way that it has lax limits defined in the same way as in Cat 9]. (Lax limit is given as the category whose objects and morphisms are lax cones from a point or an arrow to a diagram with the topology induced from the product; by "lax", we always mean "up to coherences which are iso".)

Next, one may discuss lax monads in TCat, which, in our definition, are lax functors $C: T C a t \rightarrow T C a t$ with lax natural transformations

$$
\mu: C C \rightarrow C, \eta: I d \rightarrow C
$$

which are associative and unital up to coherence isos with commutative coherence diagram same as for lax monoids. For a lax monad $C$ in TCat we then have a category of lax $C$-algebras whose objects are objects $M$ of $T C a t$ together with a functor

$$
\theta: C M \rightarrow C
$$

satisfying associativity and unitality up to coherence isos with commutative coherence diagrams of the same form as those for categories with lax action of a lax monoid.

Then lax algebras over a lax monad in TCat form a 2-category which has lax limits created by the forgetful functor to TCat. We may be interested in lax algebras over a strict monad, for example the monad associated with a theory $T$. One example of a lax monad whose lax algebras we are interested in is gotten from a 2-theory $(\Theta, T)$ and a lax $T$-algebra $I$. Then we can define a lax monad $C_{\Theta, I}$ not 
over TCat, but over the category $T c a t^{I^{k}}$ of strict functors $I^{k} \rightarrow T C a t$. In effect, $C_{\Theta, I}(X)$ has

$$
C_{\Theta, I}(X)_{i}=\coprod \Theta(m)\left(\left(\gamma_{1}, \ldots \gamma_{p}\right) ; \gamma\right)
$$

where the coproduct is indexed over $\left(j_{1}, \ldots j_{m}\right) \in I, \gamma \in T(m)^{k}, \gamma\left(j_{1}, \ldots j_{m}\right)=i$, $\gamma_{1}, \ldots \gamma_{p} \in T(m)^{k}$. Then lax $C_{\Theta, I}$-algebras are precisely lax algebras over $(\Theta, T)$ with underlying lax $T$-algebra $I$.

We are now ready to describe a topological lax semiring $\hat{\mathbb{C}}_{2}$ with an object -1 such that $1 \oplus(-1)$ is in the same connected component as 0 . First consider the lax semiring $s \mathbb{C}_{2}$ of pairs $\left(V_{+}, V_{-}\right), V_{+}, V_{-} \in \operatorname{Obj}\left(\mathbb{C}_{2}\right)$ with the lax $\mathbb{C}_{2}$-module structure given by $\mathbb{C}_{2} \oplus \mathbb{C}_{2}$ and multiplication

$$
\left(V_{+}, V_{-}\right) \otimes\left(W_{+}, W_{-}\right)=\left(V_{+} \otimes W_{+} \oplus V_{-} \otimes W_{-}, V_{+} \otimes W_{-} \oplus V_{-} \otimes W_{+}\right) .
$$

Now in $s \mathbb{C}_{2}$, consider the full subcategory $J$ on pairs $\left(V_{+}, V_{-}\right)$where $\operatorname{dim}\left(V_{+}\right)=$ $\operatorname{dim}\left(V_{-}\right)$. Then $J$ is a lax $s \mathbb{C}_{2}$-module, and $J \oplus \mathbb{C}_{2}$ is a lax commutative $\mathbb{C}_{2}$-algebra with a lax commutative $\mathbb{C}_{2}$-algebra morphisms $J \oplus C_{2} \rightarrow \mathbb{C}_{2}$ (an augmentation) and $J \oplus \mathbb{C}_{2} \rightarrow s \mathbb{C}_{2}$ (the inclusion). Thus, we have a lax simplicial commutative $\mathbb{C}_{2}$-algebra (=lax functor $\Delta^{O p} \rightarrow$ lax commutative $\mathbb{C}_{2}$-algebras)

$$
B_{\mathbb{C}_{2}}\left(\mathbb{C}_{2}, J \oplus \mathbb{C}_{2}, s \mathbb{C}_{2}\right) .
$$

We propose $\hat{\mathbb{C}}_{2}$ to be the realization of (30). This needs some explaining, namely we must define realization. We shall describe a lax realization functor

$$
\mathcal{A} \text {. } \rightarrow|\mathcal{A}|
$$

from lax simplicial commutative $\mathbb{C}_{2}$-algebras to topological commutative $\mathbb{C}_{2}$-algebras (i.e. commutative $\mathbb{C}_{2}$-algebras in $T C a t$ ). We want to mimic as closely as possible the strict construction. This means that we will define (30) as the lax simplicial realization in the 2 -category of lax $\mathbb{C}_{2}$-modules, which we must define. First, let, for a space $X, \mathbb{C}_{2} X$ be the free lax $\mathbb{C}_{2}$-module on $X$ (objects and morphisms are finite formal linear combinations with coefficients in objects and morphisms of $\mathbb{C}_{2}$, and the topology is induced from the topologies of finite powers of $X$ ). We want the realization $|\mathcal{A}|$ to be the lax coequalizer of

$$
\underset{m, n}{\oplus}\left(\mathbb{C}_{2} \Delta_{m} \otimes \mathcal{A}_{n}\right) \underset{n}{\rightarrow} \underset{n}{\rightarrow}\left(\mathbb{C}_{2} \Delta_{n} \otimes \mathcal{A}_{n}\right)
$$

(the arrows are the usual two arrows coming from lax simplicial structure, $\oplus, \otimes$ are over $\mathbb{C}_{2}$ ). To construct the lax coequalizer (31), we can take the objects of

$$
\underset{m, n}{\oplus}\left(\mathbb{C}_{2} \Delta_{m} \otimes \mathcal{A}_{n}\right) \oplus \underset{n}{\oplus}\left(\mathbb{C}_{2} \Delta_{n} \otimes \mathcal{A}_{n}\right) .
$$

To get morphisms, we take the morphisms of (32), and adjoin isomorphisms between all source and target objects of the arrows in (31). Take the free topological category spanned by these morphisms, modulo the obvious commutative diagrams required. This gives us a category with the lax $\mathbb{C}_{2}$-module (32) as a subcategory. The free construction we must then perform is applying the strict left adjoint to the forgetful functor from the category of lax $\mathbb{C}_{2}$ modules with lax submodule (32) on the same set of objects (taking only functors which are identity on objects) to the category 
of categories with subcategory (32) on the same set of objects (taking only functors which are identity on objects). As usual, the functors are strict because objects and coherences are already specified.

This completes the construction of the lax simplicial realization (30). One must still prove that this is a lax $\mathbb{C}_{2}$-algebra, but this is accomplished analogously as in the strict case, using the shuffle map (and the morphism definition (27) to assure continuity).

Now topological SLCMC's $C(\mathcal{M}), C(\mathcal{M}, H)$ for a finitely generated free topological lax $\hat{\mathbb{C}}_{2}$-module $\mathcal{M}$ are defined analogously as over $\mathbb{C}_{2}$. (Since the underlying stack of covering spaces $I=$ Set does not change, LCMC's can be described as lax algebras over a lax monad in TCat as above, and therefore stacks over a Grothendieck topology can be defined to be, as usual, contravariant functors which take Grothendieck covers to lax limits.)

However, the topology would be of little use if we simply took for our definition of modular functor a lax morphism of SLCMC's from $\mathcal{C}$ to $C(\mathcal{M})$ (similarly for CFT's). Instead, the corresponding "derived notion" is appropriate. This means that we should consider lax morphisms of topological SLCMC's

$$
B\left(C_{\Theta, S}, C_{\Theta, S}, \mathcal{C}\right) \rightarrow C(\mathcal{M})
$$

where $\Theta$ denotes the 2-theory of LCMC's, and $S$ the lax commutative monoid of finite sets, as above. The left hand side is obtained by taking the bar construction section-wise and then applying the lax left adjoint to the forgetful functor from SLCMC's to pre-stacks of LCMC's.

It still remains to define a realization functor from lax simplicial lax $C_{\Theta, S^{-}}$ algebras to topological lax $C_{\Theta, S}$-algebras. Analogously as in the case of $B_{\mathbb{C}_{2}}$, however, we may simply lax-realize in the 2-category $(\mathrm{TCat})^{S e t^{2}}$ (where it is easy to construct lax colimits, cf. 9]), and use the lax analogue of Milnor's map $|A| \times|B| \rightarrow|A \times B|$ to obtain lax $C_{\Theta, S}$-algebra structure on the realization. We omit the details.

\section{The general anomaly for open-Closed CFT.}

In this section, we shall apply the principles of Section 5 to propose the a general definition of open-closed CFT with both multiple $D$-branes and multi-dimensional conformal anomaly (although without any group completion). We shall see, however, that this is necessarily even much more complicated than what we have done in Section[5] We have already argued that neither the "set of $D$-branes" nor the "set of labels" should be sets. Rather, they should be higher vector spaces. However, on a boundary component of the worldsheet where several open parametrization components are present, we need to take traces of "sets of labels" over "sets of $D$ branes". This suggests that our model of "set of $D$-branes" must be one categorical level above our notion of "set of labels".

Therefore, we propose that the "set of $D$-branes" be a 3 -vector space $\mathcal{A}$. When dealing with 3 -vector spaces, note that they are 2 -categories. 3 -vector spaces are, by definition, 2-lax modules over the 2-lax commutative semiring $\mathbb{C}_{3}$. We must, of course, define these notions. On generalizing from lax to 2-lax structures, we find it 
easiest to follow the approach of [15]. Let $T$ be a theory. Then let $T h(T)$ be the free theory on $T$, with the canonical projection of theories $\phi: T h(T) \rightarrow T$. Let $G$ be a groupoid with objects $T h(T)$ and one isomorphism $x \rightarrow y$ for every $x, y \in T h(T)$ which satisfy $\phi(x)=\phi(y)$. Then $(T h(T), G)$ is a theory (strictly) enriched over categories and a lax $T$-algebra is the same thing as a strict $(T h(T), G)$-algebra enriched over categories.

Now to go to the next level, consider the forgetful functor

$$
U: \text { Theories enriched over groupoids } \rightarrow \mathcal{P}
$$

where $\mathcal{P}$ is the (strict) category of pairs $(T, G)$ where $T$ is a theory, $G$ is a graph with objects $T$. Then let $F$ be the left adjoint of $U$. Notice that $F$ is the identity on objects $T$, so we may write

$$
F(T, G)=(T, F(G))
$$

Now we have a map of theories enriched over groupoids:

$$
\psi:(\operatorname{Th}(T), F(G)) \rightarrow(T h(T), G) .
$$

Therefore, we may create a 2-category $(T h(T), F(G), H)$ by putting exactly one 2 -isomorphism between every $\alpha, \beta \in F(G)$ with $\psi(\alpha)=\psi(\beta)$. Then the 2-category $(T h(T), F(G), H)$ is naturally a theory (strictly) enriched over 2-categories, and a 2-lax T-algebra is a 2-category which is a strict $(T h(T), F(G), H)$-algebra enriched over 2-categories. (Obviously, one may proceed further in the same way to define even higher laxness, but we shall not need that here.)

One remark to be made is that theories, strictly speaking, do not model universal algebras which are modelled on more than one set, such as module over a ring (which is modelled over two sets). However, algebras modelled over $k$ sets can be easily included in the formalism by modifying the concept of theory to a category with objects $\mathbb{N}^{k}$ (i.e. $k$-tuples of natural numbers) with the axiom that for all $a, b \in \mathbb{N}^{k}$, $a+b$ is the categorical product of $a, b$. All of our constructions generalize to this context.

Now to identify the categorical levels with the levels we considered before, we will denote objects of 3-vector spaces by $\epsilon_{0}$ and morphisms of 3-vector spaces by $\rightarrow_{0}$. Thus, a 0-morphism $\mathbb{C}_{3} \rightarrow \mathbb{C}_{3}$ (where $\mathbb{C}_{3}$ is the lax symmetric monoidal category of 2 -vector spaces) is a 2 -vector space, and the notations $\rightarrow_{1}, \rightarrow_{2}$ of such 2 -vector spaces will coincide with the notations we used above.

Now given the 3 -vector space $\mathcal{A}$ ("the set of $D$-branes"), we must introduce the "set of labels" for anomalies. The "set of closed labels" will be, as before, a 2-vector space, which we will denote by $\mathcal{C}$. The "set of open labels" will be an object of the form

$$
\mathcal{O} \in_{0} \mathcal{A} \otimes_{\mathbb{C}_{3}} \mathcal{A}^{*}
$$

(We remark here that $\mathcal{A}^{*}$ for a 3 -vector space $\mathcal{A}$ is defined analogously as in the case of 2-vector spaces.) Now we would like to define an $\operatorname{SLCMC~} C(\mathcal{A} ; \mathcal{C}, \mathcal{O})$. All our SLCMC's in this Section shall have two labels, 1 and $m$ (closed and open). However, note that there is another subtlety we must provide for, namely that the set $\Gamma$ of all possible graphs whose vertices are open and closed parametrization and $D$-brane components and edges describe their incidence relations with the obvious conditions (e.g. all vertices have degree 2 etc.) is itself an SLCMC, and in order 
to correctly keep track of incidences on the boundary, we must consider SLCMC's over $\Gamma$.

We shall only describe sections of $C(\mathcal{A} ; \mathcal{C}, \mathcal{O})$ over a given object $G$ of $\Gamma$ over a single point, over four given sets $S_{1, \text { in }}, S_{1, \text { out }}, S_{m, \text { in }}, S_{m, \text { out }}$ of inbound and outbound closed and open "components". Let $P$ denote the set of closed $D$-brane components of $G$. Before making the definition, note that we have canonical dual 0-morphisms

$$
\mathbb{C}_{3} \stackrel{\eta}{\longrightarrow} \mathcal{A} \otimes_{\mathbb{C}_{3}} \mathcal{A}^{*} \stackrel{\epsilon}{\longrightarrow} \mathbb{C}_{3} .
$$

(If no further discussion is made, (34) requires a finiteness assumption about $\mathcal{A}$.) Their composition is a 2 -vector space which we shall denote by $\operatorname{tr}_{0} \mathcal{A}$. The set of sections of $C(\mathcal{A} ; \mathcal{C}, \mathcal{O})$ are 1 -elements

$$
\begin{aligned}
& M \in_{1} \underset{S_{1, \text { in }}}{\otimes} \mathcal{C}^{*} \otimes \underset{S_{1, \text { out }}}{\otimes} \mathcal{C} \otimes \\
& \underset{P}{\otimes} t r_{0} \mathcal{A} \otimes t r_{0, \text { cyclic }}\left(\underset{S_{m, \text { in }}}{\otimes} \mathcal{O}^{*} \otimes \underset{S_{m, \text { out }}}{\otimes} \mathcal{O}\right) .
\end{aligned}
$$

Here the tensor products are over $\mathbb{C}_{2}$, and $t r_{0, \text { cyclic }}$ denotes composition with the tensor product of the appropriate number of $\epsilon$ 's; note that although not explicitly written, the definition of $t r_{0, \text { cyclic }}$ makes use of all of the structure of $G$. Now in order to give (35) a structure of LCMC, one must show an appropriate gluing property, but this is analogous to our discussion for closed CFT's.

Now let, as above, $\mathcal{B}$ be the SLCMC of closed-open worldsheet with one closed and one open label. Then an open-closed CFT anomaly (modular functor) is a map of SLCMC's over $\Gamma$

$$
\mathcal{B} \rightarrow C(\mathcal{A} ; \mathcal{C}, \mathcal{O}) .
$$

Now to define open-closed CFT, we must add the "Hilbert spaces". The "closed Hilbert space" is, as above,

$$
H \in_{1} \mathcal{C}^{\text {Hilb }} .
$$

The "open Hilbert space" is a 1-morphism

$$
K: \eta \rightarrow_{1} \mathcal{O}^{H i l b}
$$

where $\eta$ is as in (34). We shall now define an $\operatorname{SLCMC~} C(\mathcal{A} ; \mathcal{O}, \mathcal{C} ; H, K)$ over $\Gamma$. As above, we shall specialize to sections over a single point and single object of $\Gamma$, with the same notation as above. Then a section consists of a section of $C(\mathcal{A} ; \mathcal{C}, \mathcal{O})$ and a 2-morphism

$$
\begin{aligned}
& M \rightarrow_{2} \underset{S_{1, \text { in }}}{\hat{\otimes}} H^{*} \hat{\otimes} \underset{S_{1, \text { out }}}{\otimes} H \hat{\otimes} \otimes \underset{P}{\otimes} \eta_{t r_{0} \mathcal{A}} \\
& \hat{\otimes} t r_{1, \text { cyclic }}\left(\underset{S_{m, \text { in }}}{\hat{\otimes}} K^{*} \hat{\otimes} \underset{S_{m, \text { out }}}{\otimes} K\right) .
\end{aligned}
$$


Here $t r_{1, c y c l i c}$ is given by the structure of 2 -category, and $\eta_{t r_{0} \mathcal{A}}$ is the canonical "unit" 1-element of $\operatorname{tr}_{0} \mathcal{A}$. To be more precise, write, in (34),

$$
\eta\left(\mathbb{C}_{2}\right)=\bigoplus_{i=1}^{n} \mathcal{V}_{i} \otimes_{\mathbb{C}_{2}} \phi_{i}
$$

so

$$
\operatorname{tr}_{0} \mathcal{A}=\bigoplus_{i=1}^{n} \phi_{i} \mathcal{V}_{i}
$$

But then one can show

$$
\phi_{i} \mathcal{V}_{j}=\delta_{i}^{j} \mathbb{C}_{2}
$$

so we have

$$
\operatorname{tr}_{0} \mathcal{A}=\bigoplus_{i=1}^{n} \mathbb{C}_{2}
$$

and we can write

$$
\eta_{t r_{0} \mathcal{A}}=\bigoplus_{i=1}^{n} \mathbb{C} \in_{1} \bigoplus_{i=1}^{n} \mathbb{C}_{2} .
$$

Of course, such discussion reveals the weaknesses of the higher vector space formalism, and the desirability to really work, again, in a suitable higher group completion. However, we do not work out that approach here.

\section{Appendix: Stacks of lax COMmutative monoids With CANCELlation}

To make this paper self-contained, we review here the basic definitions [14 related to stacks of lax commutative monoids with cancellation (SLCMC's). We must begin by defining lax algebras. The formalism we use is theories accoring to Lawvere, and their extension which we call 2-theories. Recall first that a theory according to Lawvere [17] is a category $T$ with objects $\mathbb{N}$ (the set of all natural numbers $0,1,2, \ldots)$ such that $n$ is the product of $n$ copies of 1 . Categories of algebraic structures given by a set of operations and relations on one set $X$ can be encoded by a theory, where $T_{n}=\operatorname{Hom}(n, 1)$ is the set of all $n$-ary operations of the algebraic structure (including all possible compositions, repetitions of one or more variables, etc.).

Definition: A 2-theory consists of a natural number $k$, a theory $T$ and a (strict) contravariant functor $\Theta$ from $T$ to the category of categories (and functors) with the following properties. Let $T^{k}$ be a category with the same objects as $T$, and $H_{o m}^{T^{k}}(m, n)=\operatorname{Hom}_{T}(m, n)^{\times k}$ (obvious composition). Then

$$
\operatorname{Obj}(\Theta(m))=\coprod_{n} \operatorname{Hom}_{T^{k}}(m, n),
$$

on morphisms, $\Theta$ is given by precomposition on $\operatorname{Obj}(\Theta(m))$, and $\gamma \in \operatorname{Hom}_{T^{k}}(m, n)$ is the product, in $\Theta(m)$, of the $n$-tuple $\gamma_{1}, \ldots, \gamma_{n} \in \operatorname{Hom}_{T^{k}}(m, 1)$ with which it is 
identified by the fact that $T$ is a theory. We also speak of a 2-theory fibered over the theory $T$.

The example relevant to CFT is the 2-theory of commutative monoids with cancellation. $T$ is the theory of commutative monoids with an operation + , and $k=2$. The 2-theory $\Theta$ has three generating operations, addition (or disjoint union) $+: X_{a, c} \times X_{b, d} \rightarrow X_{a+b, c+d}$, unit $0 \in X_{0,0}$ and cancellation (or gluing) $\check{?}: X_{a+c, b+c} \rightarrow X_{a, b}$. The axioms are commutativity, associativity and unitality for

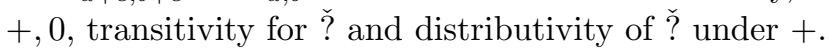

To get further, one needs to define algebras and lax algebras over theories and 2-theories. An algebra over a theory $T$ is a set $I$ together with, for each $\gamma \in T_{n}$, a map $\bar{\gamma}: I^{\times n} \rightarrow I$, satisfying appropriate axioms. These axioms can be written out explicitly, but a quick way to encode them is to notice that for a set $I$, we have the endomorphism theory $\operatorname{End}(I)$ where $\operatorname{End}(I)(n)=M a p\left(I^{\times n}, I\right)$, and we may simply say that a structure of $T$-algebra on $I$ is given by a map of theories $T \rightarrow \operatorname{End}(I)$.

To define an algebra over a 2-theory $\Theta$ fibered over a theory $T$, we must first have an algebra $I$ over the theory $T$ (the 'indexing theory'). This gives us, for $\gamma \in H_{T^{k}}(m, 1)$, a $k$-tuple of maps $\bar{\gamma}: I^{\times m} \rightarrow I$. In an algebra over the 2-theory, we have, in addition, a map

$$
X: I^{\times k} \rightarrow \text { Sets. }
$$

For a morphism in $\phi \in M o r(\Theta)$ from $\left(\gamma_{1}, \ldots, \gamma_{n}\right) \in \operatorname{Hom}_{T^{k}}(m, n)$ to $\gamma \in \operatorname{Hom}_{T^{k}}(m, 1)$, we have, for each choice $i_{1}, \ldots, i_{m}$ of elements of $I$, maps

$$
\bar{\phi}: X\left(\overline{w_{1}}\left(i_{1}, \ldots, i_{m}\right)\right) \times \ldots \times X\left(\overline{w_{n}}\left(i_{1}, \ldots, i_{m}\right)\right) \rightarrow X\left(\bar{w}\left(i_{1}, \ldots, i_{m}\right)\right),
$$

satisfying appropriate axioms. Once again, we can avoid writing them down explicitly by defining the endomorphism 2 -theory. Consider a set $I$ and a map

$$
X: I^{k} \rightarrow \text { Sets. }
$$

To such data there is assigned a 2-theory $\operatorname{End}(X)$ fibered over the theory $\operatorname{End}(I)$ : let

$$
\Theta\left(w ; w_{1}, \ldots, w_{n}\right)
$$

consist of the set of all possible simultaneous choices of maps

$$
X\left(w_{1}\left(i_{1}, \ldots, i_{m}\right)\right) \times \ldots \times X\left(w_{n}\left(i_{1}, \ldots, i_{m}\right)\right) \rightarrow X\left(w\left(i_{1}, \ldots, i_{m}\right)\right)
$$

where $i_{j}$ range over elements of $I$. A structure of an algebra over the 2-theory $\Theta$ fibered over $T$ is given by a morphism of 2 -theories

$$
(\Theta, T) \rightarrow(\operatorname{End}(X), \operatorname{End}(I)) .
$$

A lax algebra over a theory is a category $I$, with maps $\bar{\gamma}$ which are functors. We do not, however, require that these maps define a strict morphism from $T$ to the endomorphism theory of $I$. Instead, this is only true up to certain natural isomorphisms, which we call coherence isomorphisms, which in turn are required to satisfy certain commutative diagrams, which are called coherence diagrams. This is, of course, always the case when defining lax algebras of any kind. But now the benefit of introducing theories is that the coherences and coherence diagrams always have the same shape. To be more precise, recall that the notion of theory itself is an 
algebraic structure which can be encoded by the sequence of sets $T(n)$, and certain operations on these sets satisfying certain identities. Denoting the set of operations defining theories by $G$ (for 'generators'), and identities by $R$ (for 'relations'), we observe that the set of coherence isomorphisms we must require for lax $T$-algebras is always in bijective correspondence with $G$, while the set of coherence diagrams needed is in bijective correspondence with $R$ !

The concept of lax algebra over a 2-theory is defined in a similar fashion, but one important point is that one doesn't want to consider the most general possible type of laxness (since that would lead to a 3-category). Rather, one starts with a lax algebra $I$ over the indexing theory, and a strict functor

$$
X: I^{\times k} \rightarrow \text { Categories; }
$$

appropriate coherence isomorphisms and diagrams then follow in the same way as in the case of lax algebras over a theory (are indexed by operations and identities of 2-theories interpreted as a 'universal algebras' - see [14).

The lax commutative monoid we most frequently consider is the groupoid $S$ of finite sets and isomorphisms (the operation is disjoint union). More generally, we often consider a set of labels $K$ and the lax commutative monoid of $S_{K}$ of sets $A$ labelled by $K$, i.e. maps $A \rightarrow K$. Again, the operation is disjoint union. The example of lax commutative monoid with cancellation fibered over $S$ considered in [14] is the groupoid $\mathcal{C}$ of worldsheets or rigged surfaces. These are 2-dimensional smooth manifolds with smooth boundary; further, each boundary component is parametrized by a smooth diffeomorphism with $S^{1}$, and the surface has a complex structure with respect to which the boundary parametrization is analytic. Morphisms are biholomorphic diffeomorphisms commuting with boundary parametrization. Addition is disjoint union, and cancellation is gluing of boundary components. Similarly, again, one can consider the LCMC $\mathcal{C}_{K}$ of worldsheets with $K$-labelled boundary components, which is an LCMC over $S_{K}$.

To complete the picture, one needs to consider stacks. We note that lax algebras over a theory and lax algebras (in our sense) over a 2-theory form 2-categories which have lax limits of strict diagrams (see Fiore [9]). For older references, which however work in slightly different contexts (and with different terminology), see Borceux [3] or 9]. For the 2-category structure, 1-morphisms are lax morphisms of lax algebras (functors such that there is a natural coherence isomorphism for every element of $G$ ), and 2-morphisms are natural isomorphisms which commute with the operations given by the theory (or 2-theory). Now for any 2-category $\mathcal{C}$ with lax limits, and every Grothendieck topology $\mathcal{B}$, we can define $\mathcal{B}$-stacks over $\mathcal{C}$ : they are simply contravariant functors $\mathcal{B} \rightarrow \mathcal{C}$ which turn Grothendieck covers into lax limits. Note that such stacks then themselves form a 2-category with respect to stack versions of the same 1-morphisms and 2-morphisms.

Now to turn $\mathcal{C}$ into a stack of lax commutative monoids with cancellation, we must first specify the Grothendieck topology. Note that there are two choices of the topology, either just (finite-dimensional) smooth manifolds and open covers (non-chiral setting) or finite-dimensional complex manifolds and open covers (chiral setting). As remarked in Section 2 above, however, $D$-branes can only be considered in the non-chiral setting. To define the stack, one must first define the underlying 
stack of lax commutative monoids; the answer is simply the stack of covering spaces with finitely many sheets. Now one must define smooth or holomorphic families of worldsheets. We shall only make the definition in the holomorphic case, the smooth case is analogous. The most convenient way to make this precise is to consider, for a worldsheet $X$, the complex 1-manifold $Y$ obtained by gluing, locally, solid cylinders to the boundary components of $X$. Then, a holomorphic family of rigged surfaces $X$ over a finite dimensional complex manifold $B$ is a holomorphic map

$$
q: Y \rightarrow B
$$

transverse to every point, such that $\operatorname{dim}(Y)=\operatorname{dim}(B)+1$ and $B$ is covered by open sets $U_{i}$ for each of which there are given holomorphic regular inclusions

$$
s_{i, c}: D \times U_{i} \rightarrow Y
$$

with

$$
q \circ s_{i, c}=I d_{U_{i}}
$$

where $c$ runs through some indexing set $C_{i}$. Further, if $U_{i} \cap U_{j} \neq \emptyset$, we require that there be a bijection $\iota: C_{i} \rightarrow C_{j}$ such that

$$
\left.s_{i, c}\right|_{D \times\left(U_{i} \cap U j\right)}=\left.s_{j, \iota(c)}\right|_{D \times\left(U_{i} \cap U_{j}\right)} .
$$

Then we let

$$
X=Y-\left(\bigcup_{i} \bigcup_{c \in C_{i}} s_{i, c}\left(\left(D-S^{1}\right) \times U_{i}\right)\right)
$$

Then the fiber of $X$ over each $b \in B$ is a rigged surface, which vary holomorphically in $b$, in the sense we want. (Note that the reason the maps $s_{c}$ cannot be defined globally in $B$ is that it is possible for a non-trivial loop in $\pi_{1}(B)$ to permute the boundary components of $X$.) The treatment of $\mathcal{C}_{K}$ is analogous. As a rule, we shall use the same symbol for the SLCMC's $\mathcal{C}, \mathcal{C}_{K}$ as for the corresponding LCMC's (their sections over a point).

We are done with the review of SLCMC's, but we shall still briefly cover CFT's, as defined in 14. Although this definition is subsumed by Section 5 above, the reader might still find the more elementary definition useful while reading the earlier sections. Let $\mathcal{H}_{1}, \ldots, \mathcal{H}_{n}$ be complex (separable) Hilbert spaces. Then on $\mathcal{H}_{1} \otimes \ldots \otimes$ $\mathcal{H}_{n}$, there is a natural inner product

$$
\left\langle a_{1} \otimes \ldots \otimes a_{n}, b_{1} \otimes \ldots \otimes b_{n}\right\rangle=\left\langle a_{1}, b_{1}\right\rangle\left\langle a_{2}, b_{2}\right\rangle \ldots\left\langle a_{n}, b_{n}\right\rangle .
$$

The Hilbert completion of this inner product space is called the Hilbert tensor product

$$
\mathcal{H}_{1} \hat{\otimes} \ldots \hat{\otimes} \mathcal{H}_{n} .
$$

Now an element of (39) is called trace class if there exist unit vectors $e_{i j} \in \mathcal{H}$ where $j=1, . ., n$ and $i$ runs through some countable indexing set $I$ such that

$$
x=\sum_{i \in I} \mu_{i}\left(e_{i 1} \otimes \ldots \otimes e_{i n}\right)
$$

and

$$
\sum_{i \in I}\left|\mu_{i}\right|<\infty
$$


The vector subspace of (39) of vectors of trace class will be denoted by

$$
\mathcal{H}_{1} \otimes \ldots \otimes \mathcal{H}_{n}
$$

Note that (40) is not a Hilbert space. We have, however, canonical maps

$$
\otimes:\left(\mathcal{H}_{1} \otimes \ldots \otimes \mathcal{H}_{n}\right) \otimes\left(\mathcal{H}_{n+1} \otimes \ldots \otimes \mathcal{H}_{m+n}\right) \rightarrow \mathcal{H}_{1} \otimes \ldots \otimes \mathcal{H}_{m+n}
$$

and, if $\mathcal{H}^{*}$ denotes the dual Hilbert space to a complex Hilbert space $\mathcal{H}$,

$$
\operatorname{tr}: \mathcal{H} \otimes \mathcal{H}^{*} \otimes \mathcal{H}_{1} \otimes \ldots \otimes \mathcal{H}_{n} \rightarrow \mathcal{H}_{1} \otimes \ldots \otimes \mathcal{H}_{n} .
$$

This allows us to define a particular example of stack of LCMC's based on $\mathcal{H}$, which we will call $\underline{\mathcal{H}}$. The underlying stack of lax commutative monoids (T-algebras) is $S$. Now let $B \in \mathcal{B}$. Let $s, t$ be sections of the stack $S$ over $B$, i.e. covering spaces of $B$ with finitely many sheets. Then we have an infinite-dimensional holomorphic bundle over $B$

$$
\left(\mathcal{H}^{*}\right)^{\bigotimes s} \otimes \mathcal{H}^{\bigotimes t} .
$$

What we mean by that is that there is a well defined sheaf of holomorphic sections of (41) (note that it suffices to understand the case when $s, t$ are constant covering spaces, which is obvious). Now a section of $\underline{\mathcal{H}}$ over a pair of sections $s, t$ of $S$ is a global section of (41) over $b$; the only automorphisms of these sections covering $I d_{s} \times I d_{t}$ are identities. The operation,$+ \check{?}$ are given by the operations $\otimes, \operatorname{tr}$ (see above).

We can also define a variation of this LCMC for the case of labels indexed over a finite set $K$. We need a collection of Hilbert spaces

$$
\mathcal{H}_{K}=\left\{\mathcal{H}_{k} \mid k \in K\right\} \text {. }
$$

Then we shall define a stack of LCMC's $\underline{\mathcal{H}}_{K}$. The underlying stack of $T$-algebras (commutative monoids) is $S_{K}$. Let $s, t$ be sections of $S_{K}$ over $B \in \mathcal{B}$. The place of (41) is taken by

$$
\left(\mathcal{H}_{K}^{*}\right)^{\bigotimes s} \otimes \mathcal{H}_{K}^{\bigotimes t}
$$

By the sheaf of holomorphic section of (42) when $B$ is a point we mean that $\nabla$ powers of $\mathcal{H}_{k}$ (or $\mathcal{H}_{k}^{*}$ ) for each label $k \in K$ are taken according to the the number of points of $\Gamma(t)(\operatorname{resp} . \Gamma(s)$ ); when $s$ and $t$ are constant covering spaces $B$, the space of sections of (42) is simply the set of holomorphically varied elements of the spaces of sections over points of $B$ (which are identified). This is generalized to the case of general $s, t$ in the obvious way (using functoriality with respect to permutations of coordinates). As above, the only automorphisms of these sections covering $I d_{s} \times I d_{t}$ are identities.

Remark: For technical reasons (different types of convergence), the above setup involving Hilbert spaces and trace class elements is sometimes insufficient (see Example below and Section 4 above). Because of that, it is beneficial to generalize to a context where $\underline{\mathcal{H}}$ simply means any SLCMC over $S$ (resp. $S_{K}$ in the labelled case) whose spaces of sections are vector spaces, the operation ? is linear, and the operation + is bilinear. We shall further assume that $\underline{\mathcal{H}}$ is a sheaf in the sense that the only endomorphisms over the identity in $S$ (resp. $S_{K}$ ) is the identity.

Example: Consider the free bosonic CFT (14) discussed in Section 4 As remarked above, the description 14 is actually already not quite right: the vacuum state is 
to be an eigenstate of momentum 0 , but there is no non-zero function in $L^{2}(\mathbb{R}, \mathbb{C})$ with support in the set $\{0\}$. For the same reason, we also find that the operator $U_{A_{q}}$ associated with the standard annulus $A_{q}$ is not trace class as defined (since, for example, 1 is a limit point of the spectrum of $U_{A_{q}}$ ). This is the usual problem in quantum mechanics. In the present setting, a solution along the lines of the Remark can be obtained as follows: Let $\mathcal{F}$ be the bosonic Fock space, i.e.

$$
\mathcal{F}=\widehat{\operatorname{Sym}}\left\langle z^{n}, \bar{z}^{n}, n>0\right\rangle .
$$

Then the sections of $\underline{\mathcal{H}}$ over $(s, t)$ (over a point) are elements

$$
f \in \prod_{k \in \mathbb{R}^{|s|+|t|}} \mathcal{F}^{\hat{\otimes}|t|} \hat{\otimes} \mathcal{F}^{* \hat{\otimes}|s|}
$$

(the product is categorical product of vector spaces) which have the property that for every pair of injections $i: u \rightarrow s, j: u \rightarrow t$ and every map

$$
k:(s-i(u)) \coprod(t-j(u)) \rightarrow \mathbb{R},
$$

we have

$$
\int_{x \in \mathbb{R}^{u}} \mu\left(\phi_{i, j, k}(x)\right)<\infty
$$

where $\phi_{i, j, k}(x)=f(y), y \in \mathbb{R}^{s} \amalg^{t}$ is defined by $y(i(r))=y(j(r))=x(r), y(r)=k(r)$ for $r \in(s-i(u)) \amalg(t-j(u))$ and, for $z \in \mathcal{F}^{\hat{\otimes}|t|} \hat{\otimes} \mathcal{F}^{* \hat{\otimes}|s|}$,

$$
\mu(z)=\inf \left(\left\{\sum\left|a_{i}\right| \mid z=\sum a_{i} \underset{x \in s \amalg t}{\otimes} e_{x},\left\|e_{x}\right\|=1 \text { for all } x\right\},\right.
$$

and gluing along $i, j$ is defined by

$$
\check{f}(k)=\int_{x \in \mathbb{R}^{u}} \operatorname{tr}\left(\phi_{i, j, k}(x)\right) .
$$

The expression (43) is always defined because of the condition imposed, and the condition is preserved by the gluing operation by Fubini's theorem.

Now we can define an abstract $C F T$ based on an SLCMC $\mathcal{D}$ with underlying stack of lax commutative monoids (SLCM) $S$ simply as a 1-morphism of SLCMC's, over $I d_{S}$,

$$
\mathcal{D} \rightarrow \underline{\mathcal{H}} .
$$

A similar definition applies if $\mathcal{D}$ has underlying SLCM $S_{K}$, with $\underline{\mathcal{H}}$ replaced by $\underline{\mathcal{H}}_{K}$.

However, this notion still is not definitive in the sense that it does not capture anomaly. In Section 5 above, we give the most general definition of modular functor, but it is useful to review a direct definition from [14 at least in one special case, namely 1-dimensional anomaly. To this end, we give the definition of $\mathbb{C}^{\times}$-central extension (or, equivalently, 1-dimensional modular functor) on an LCMC $\mathcal{D}$. This is a strict morphism of stacks of LCMC's

$$
\psi: \tilde{\mathcal{D}} \rightarrow \mathcal{D}
$$


over $I d$ on the underlying stacks of LCM's with the following additional structure (for simplicity, let us just work in the holomorphic (chiral) setting): For each object $B$ of $\mathcal{B}$, and each pair of sections $s, t$ of $S$ over $B$, and each section $\alpha$ of $\mathcal{D}$ over $s, t, B, B^{\prime} \rightarrow B$,

$$
\psi^{-1}\left(\left.\alpha\right|_{B^{\prime}}\right)
$$

with varying $B^{\prime}$ is the space of sections of a complex holomorphic line bundle over $B$. Furthermore, functoriality maps supplied by the structure of stack of LCMC's on $\tilde{D}$ are linear maps on these holomorphic line bundles. Regarding the operation + , we require that the map induced by +

$$
\psi^{-1}\left(\left.\alpha\right|_{B^{\prime}}\right) \times \psi^{-1}\left(\left.\beta\right|_{B^{\prime}}\right) \rightarrow \psi^{-1}\left(\left.(\alpha+\beta)\right|_{B^{\prime}}\right)
$$

be a bilinear map, which induces an isomorphism of holomorphic line bundles

$$
\psi^{-1}\left(\left.\alpha\right|_{B^{\prime}}\right) \otimes_{\mathcal{O}_{B^{\prime}}} \psi^{-1}\left(\left.\beta\right|_{B^{\prime}}\right) \rightarrow \psi^{-1}\left(\left.(\alpha+\beta)\right|_{B^{\prime}}\right)
$$

$\left(\mathcal{O}_{B}\right.$ is the holomorphic structure sheaf on $\left.B\right)$.

Regarding the operation ?, we simply require that if $\alpha$ is a section of $\mathcal{D}$ over $s+u, t+u, B$ where $u$ is another section of $S$ over $B$, and $\check{\alpha}$ is the section over $s, t$, $B$ which is obtained by applying the operation ? to $\alpha$, then the map of holomorphic line bundles coming from LCMC structure

$$
\psi^{-1}\left(\left.\alpha\right|_{B^{\prime}}\right) \rightarrow \psi^{-1}\left(\left.\check{\alpha}\right|_{B^{\prime}}\right)
$$

$\left(B^{\prime} \rightarrow B\right)$ be an isomorphism of holomorphic line bundles.

By a CFT with 1-dimensional modular functor over $\mathcal{D}$ with underlying stack $S$ we shall mean a CFT

$$
\phi: \tilde{D} \rightarrow \underline{\mathcal{H}}
$$

(where $\tilde{\mathcal{D}}$ is a $\mathbb{C}^{\times}$-central extension of $\mathcal{D}$ which has the property that $\phi$ is a linear map on the spaces of sections (45). Similarly in case $\mathcal{D}$ has underlying stack $S_{K}$; we simply replace $S$ by $S_{K}$ everywhere throughout the definition.

It is appropriate to comment on a weaker kind of morphism of lax algebras where we do not require that the coherence maps be iso. By a pseudomorphism of lax $T$-algebras (and similarly in the cases of lax $\Xi, T$-algebra and their stacks) we shall mean a functor

$$
f: X \rightarrow Y
$$

together with morphisms (called cross-morphism, not necessarily iso)

$$
\gamma(f, \ldots, f) \rightarrow f \gamma
$$

which commute with all the coherences in the lax $T$-algebra sense (we shall refer to these required commutative diagrams as cross-diagrams).

Remark: Although the anomaly of the linear $\sigma$-model considered in Section 4 is 1-dimensional, in Section [5 we considered higher-dimensional anomalies. It is therefore appropriate to reconcile the above remark concerning generalizing the SLCMC $\underline{\mathcal{H}}$ to cases when the Hibert/trace class model fails due to non-convergence with our discussion of higher-dimensional modular functors via 2-vector spaces. In other words, what is the right generalization of $H \in \operatorname{Obj}\left(\mathcal{M}^{H i l b}\right)$ in $C(\mathcal{M}, H)$ ? The main point is that the Hilbert tensor powers $H^{\hat{\otimes} t} \hat{\otimes} H^{* \hat{\otimes} s}$ should be replaced by a 
"vector space indexed over $\mathcal{M}$ " which depends only on $s, t$ and have appropriate designated "trace maps".

The category $\mathcal{M}^{\text {Vect }}$ of vector spaces indexed over $\mathcal{M}$ is defined as

$$
\mathcal{M} \otimes \mathbb{C}_{2} \mathbb{C}_{2}^{V e c t}
$$

where $\mathbb{C}_{2}^{\text {Vect }}$ is the lax commutative semiring of $\mathbb{C}$-vector spaces (not necessarily finitely dimensional).

Now $H \in \operatorname{Obj}\left(\mathcal{M}^{\text {Vect }}\right)$, we may consider a pseudomorphism of SLCMC's over $S$ (see above)

$$
h: S^{2} \rightarrow C\left(\mathcal{M}^{\text {Vect }}\right) .
$$

Here by $C\left(\mathcal{M}^{V e c t}\right)$ we mean the analogous construction as $C(\mathcal{M})$, but with the duals taken over $\mathbb{C}^{\text {Vect }}$, so $\left(\mathcal{M}^{\text {Vect }}\right)^{*}={ }_{\text {def }}\left(\mathcal{M}^{*}\right)^{\text {Vect }}$. Then, an $\operatorname{SLCMC~} C(\mathcal{M}, h)$ is defined as follows. Sections over a point over $\sigma \in \operatorname{Obj}(S)^{2}$ consist of a section $M$ of $C(\mathcal{M})$ over $\sigma$ and a 2-morphism

$$
M \rightarrow h(\sigma) .
$$

Stacking, and the necessary verifications, are completed in the usual way.

\section{REFERENCES}

[1] M. Ando: Power operations in elliptic cohomology and representations of loop groups, Trans. AMS 352 (2000) 5619-5666

[2] N.A.Baas, B.I.Dundas, J.Rognes: Two-vector bundles and forms of elliptic cohomology, to appear

[3] F.Borceux: Handbook of categorical algebra 1-2, Encyclopedia of Mathematics and its Applications 50-52, Cambridge University Press

[4] R.E.Borcherds: Monstrous moonshine and monstrous Lie superalgebra, Invent. Math. 109 (1992) 405-444

[5] deHoeker: String theory, in Quantum fields and strings: a course for mathematicians, vol. 2, AMS and IAS 1999, 807-1012

[6] P.Deligne, D.Freed: Notes on Supersymmetry (following J. Bernstein) in: Quantum fields and strings, a course for mathematicians, vol. 1, AMS, 1999, 41-98

[7] D.E.Diaconescu: Enhanced D-brane categories from string field theory, JHEP 0106 (2001) 16

[8] M.R.Douglas: D-branes, categories and $N=1$ SUSY, J.Math.Phys. 42 (2001) 2818-2843

[9] T.Fiore: Lax limits, lax adjoints and lax algebras, preprint, 2003

[10] I.Frenkel: Vertex algebras and algebraic curves, Seminaire Bourbaki 1999-2000, Asterisque 276 (2002) 299-339

[11] I.Frenkel, J.Lepowsky, A.Meurman: Vertex operator algebras and the monster, Pure and applied Mathematics, vol. 134, Academic Press, 1999

[12] M.B.Green, J.H.Schwartz, E.Witten: Superstring theory, vol. 1,2, Cambridge University Press, 1988

[13] P.Horava: Equivariant Topological Sigma Models, Nucl. Phys. B 418 (1994) 571-602, hep-th/9309124

[14] P.Hu, I.Kriz: Conformal field theory and elliptic cohomology, to appear in Advances in Mathematics

[15] P.Hu, I.Kriz, A.A.Voronov: On Kotsevich's Hochschild cohomology conjecture, Preprint 2001

[16] I.Kriz: On spin and modularity in conformal field theory, Ann. Sci. de ENS 36 (2003) 57-112

[17] W.F.Lawvere: Functorial semantics of algebraic theories, Proc. Nat. Acad. Sci. U.S.A. 50 $1963869-87$ 
[18] C.I.Lazaroiu: On the structure of open-closed topological field theory in two-dimensions, Nucl. Phys. B 603 (2001) 497-530

[19] C.I.Lazaroiu: Generalized complexes and string field theory, JHEP 0106 (2001) 52

[20] C.I.Lazaroiu: Unitarity, D-brane dynamics an D-brane categories, JHEP 0112 (2001) 31

[21] D.Lewellen: Sewing constraints for conformal field theories on surfaces with boundaries, Nucl. Phys. B 372 (1992) 654

[22] G.Moore: Some Comments on Branes, G-flux, and K-theory, Int. J.Mod.Phys. A16 (2001) 936-944

[23] G.Moore, N.Seiberg: Classical and Quantum Conformal Field Theory, Comm. Math. Phys. 123, (1989) 177-254

[24] G.Moore, N.Seiberg: Taming the conformal ZOO, Physics Letters B 220 (1989) 422-430

[25] G.Moore, N.Seiberg: Lectures on RCFT, Physics, Geometry and Topology, H.C.Lee, ed., (1990) 263-361

[26] J.Polchinski: String theory, Vol. 1,2, Cambridge Univ. Press, 1999

[27] A.Pressley, G.Segal: Loop groups, Oxford University Press, 1986

[28] G.Segal: Elliptic cohomology, Seminaire Bourbaki 1987/88, Asterisque 161-162 (1988) Exp. No, 695, (1989) 4, 187-201

[29] G. Segal: The definition of conformal field theory, Preprint, the 1980's

[30] G.Segal: ITP lectures, http://doug-pc.itp.ucsb.edu/online/geom99/

[31] G.Segal: Categories and cohomology theories, Topology 13 (1974), 293-312

[32] S.Stolz, P.Teichner: What is an elliptic object?, preprint, 2003

[33] R.W.Thomason: Beware the phony multiplication on Quillen's $\mathcal{A}^{-1} \mathcal{A}$, Proc. AMS 80 (1980) $4,569-573$

[34] E.Verlinde: Fusion rules and modular transformations in $2 D$ conformal field theory, Nucl. Phys. B 300 (1988) 360-376

[35] E.Witten: Overview of $K$-theory applied to strings, Int.J.Mod.Phys. A 16 (2001) 693-706 